\title{
THE MUSIC OF CLASH: PREDICTIONS ON THE CONCENTRATION-MASS RELATION
}

\author{
M. Meneghetti ${ }^{1,2,3}$, E. Rasia ${ }^{4}$, J. Vega ${ }^{5,6}$, J. Merten $^{2,7}$, M. Postman ${ }^{8}$, G. Yepes $^{5}$, F. Sembolini ${ }^{5}$, M. Donahue ${ }^{9}$, \\ S. EtToRI ${ }^{2,3}$, K. Umetsu $^{10}$, I. Balestra ${ }^{11,12}$, M. BartelmanN ${ }^{13}$, N. Benítez ${ }^{14}$, A. Biviano ${ }^{12}$, R. Bouwens ${ }^{15}$, L. Bradley ${ }^{16}$, \\ T. Broadhurst ${ }^{17,18}$, D. CoE $^{8}$, N. Czakon ${ }^{10}$, M. De Petris ${ }^{19}$, H. Ford ${ }^{16}$, C. Giocoli ${ }^{20}$, S. Gottlöber ${ }^{21}$, C. Grillo ${ }^{22}$, \\ L. Infante ${ }^{23}$, S. Jouvel ${ }^{24,25}$, D. Kelson ${ }^{26}$, A. Koekemoer ${ }^{7}$, O. Lahav ${ }^{25}$, D. Lemze ${ }^{16}$, E. Medezinski ${ }^{16}$, P. Melchior ${ }^{27}$, \\ A. Mercurio ${ }^{11}$, A. Molino ${ }^{14}$, L. Moscardini ${ }^{20}$, A. Monna ${ }^{28,29}$, J. Moustakas $^{30}$, L. A. Moustakas ${ }^{2}$, M. Nonino ${ }^{12}$,

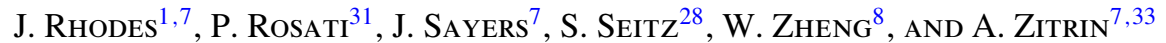 \\ ${ }^{1}$ INAF, Osservatorio Astronomico di Bologna, via Ranzani 1, I-40127 Bologna, Italy \\ 2 Jet Propulsion Laboratory, California Institute of Technology, 4800 Oak Grove Drive, Pasadena, CA 91109, USA \\ ${ }^{3}$ INFN, Sezione di Bologna, Viale Berti Pichat 6/2, I-40127 Bologna, Italy \\ ${ }^{4}$ Physics Department, University of Michigan, 450 Church Avenue, Ann Arbor, MI 48109, USA \\ ${ }^{5}$ Departamento de Fsica Terica, Universidad Autnoma de Madrid, Cantoblanco, E-28049 Madrid, Spain \\ ${ }^{6}$ LERMA, CNRS UMR 8112, Observatoire de Paris, 61 Avenue de l'Observatoire, F-75014 Paris, France \\ ${ }^{7}$ California Institute of Technology, MC 249-17, Pasadena, CA 91125, USA \\ ${ }^{8}$ Space Telescope Science Institute, 3700 San Martin Drive, Baltimore, MD 21208, USA \\ ${ }^{9}$ Department of Physics and Astronomy, Michigan State University, East Lansing, MI 48824, USA \\ ${ }^{10}$ Institute of Astronomy and Astrophysics, Academia Sinica, PO Box 23-141, Taipei 10617, Taiwan \\ ${ }^{11}$ INAF-Osservatorio Astronomico di Capodimonte, Via Moiariello 16, I-80131 Napoli, Italy \\ ${ }^{12}$ INAF/Osservatorio Astronomico di Trieste, via G. B. Tiepolo 11, I-34143 Trieste, Italy \\ ${ }^{13}$ Institut fur Theoretische Astrophysik, Universität Heidelberg, Zentrum für Astronomie, Philosophenweg 12, D-69120 Heidelberg, Germany \\ ${ }^{14}$ Instituto de Astrofísica de Andalucía (CSIC), E-18080 Granada, Spain \\ ${ }^{15}$ Leiden Observatory, Leiden University, PO Box 9513, NL-2333 Leiden, The Netherlands \\ ${ }^{16}$ Department of Physics and Astronomy, The Johns Hopkins University, 3400 North Charles Street, Baltimore, MD 21218, USA \\ ${ }^{17}$ Department of Theoretical Physics and History of Science, University of the Basque Country UPV/EHU, PO Box 644, E-48080 Bilbao, Spain \\ ${ }^{18}$ Ikerbasque, Basque Foundation for Science, Alameda Urquijo, 36-5 Plaza Bizkaia, E-48011 Bilbao, Spain \\ ${ }^{19}$ Dipartimento di Fisica, Sapienza Universit di Roma, Piazzale Aldo Moro 5, I-00185 Roma, Italy \\ ${ }^{20}$ Dipartimento di Fisica e Astronomia, Università di Bologna, Via Ranzani 2, 40127, Bologna \\ ${ }^{21}$ Leibniz-Institut für Astrophysik, An der Sternwarte 16, D-14482 Potsdam, Germany \\ 22 Dark Cosmology Centre, Niels Bohr Institute, University of Copenhagen, Juliane Maries Vej 30, DK-2100 Copenhagen, Denmark \\ ${ }^{23}$ Centro de Astro-Ingeniería, Departamento de Astronomía y Astrofísica, Pontificia Universidad Catolica de Chile, V. Mackenna 4860, Santiago, Chile \\ ${ }^{24}$ Institut de Ciéncies de l'Espai (IEEC-CSIC), E-08193 Bellaterra (Barcelona), Spain \\ ${ }^{25}$ Department of Physics and Astronomy, University College London, London WC1E 6BT, UK \\ ${ }^{26}$ Observatories of the Carnegie Institution of Washington, Pasadena, CA 91101, USA \\ ${ }^{27}$ Center for Cosmology and Astro-Particle Physics and Department of Physics, The Ohio State University, Columbus, OH 43210, USA \\ ${ }^{28}$ Universitäts-Sternwarte München, Scheinerstr. 1, D-81679 München, Germany \\ ${ }^{29}$ Max Planck Institute for Extraterrestrial Physics, Giessenbachstrasse, D-85748 Garching, Germany \\ ${ }^{30}$ Department of Physics and Astronomy, Siena College, 515 Loudon Road, Loudonville, NY 12211, USA \\ ${ }^{31}$ Dipartimento di Fisica e Scienze della Terra, Università degli Studi di Ferrara, Via Saragat 1, I-44122 Ferrara, Italy \\ ${ }^{32}$ Institute for Computational Cosmology, Durham University, South Road, Durham DH1 3LE, UK \\ Received 2014 April 10; accepted 2014 September 25; published 2014 November 21
}

\begin{abstract}
We present an analysis of the MUSIC-2 $N$-body/hydrodynamical simulations aimed at estimating the expected concentration-mass relation for the CLASH (Cluster Lensing and Supernova Survey with Hubble) cluster sample. We study nearly 1,400 halos simulated at high spatial and mass resolution. We study the shape of both their density and surface-density profiles and fit them with a variety of radial functions, including the Navarro-Frenk-White (NFW), the generalized NFW, and the Einasto density profiles. We derive concentrations and masses from these fits. We produce simulated Chandra observations of the halos, and we use them to identify objects resembling the X-ray morphologies and masses of the clusters in the CLASH X-ray-selected sample. We also derive a concentration-mass relation for strong-lensing clusters. We find that the sample of simulated halos that resembles the X-ray morphology of the CLASH clusters is composed mainly of relaxed halos, but it also contains a significant fraction of unrelaxed systems. For such a heterogeneous sample we measure an average two-dimensional concentration that is $\sim 11 \%$ higher than is found for the full sample of simulated halos. After accounting for projection and selection effects, the average NFW concentrations of CLASH clusters are expected to be intermediate between those predicted in three dimensions for relaxed and super-relaxed halos. Matching the simulations to the individual CLASH clusters on the basis of the X-ray morphology, we expect that the NFW concentrations recovered from the lensing analysis of the CLASH clusters are in the range [3-6], with an average value of 3.87 and a standard deviation of 0.61 .
\end{abstract}

Key words: dark matter - galaxies: clusters: general - gravitation lensing: weak - gravitational lensing: strong

Online-only material: color figures 


\section{INTRODUCTION}

Gravitational lensing is one the most powerful methods of investigating the distribution of matter (either dark or baryonic) in galaxy clusters. It is well known that this class of objects is particularly important in cosmology for several reasons. First, in a hierarchical model of structure formation, galaxy clusters are the most recent bound structures to form in the universe. They are often captured in the middle of violent dynamical processes like mergers between smaller structures, allowing us to study in detail how structure formation proceeds. Second, each of them is a miniature universe; their composition closely reflects the matter composition of the universe at large. Last but not least, they trace the exponential tail of the structure mass function. Tiny variations of the cosmological parameters are reflected in dramatic changes in the mass function and its evolution.

The lensing effects produced by galaxy clusters are sometimes spectacular. The light emitted by galaxies in the background of these objects interacts with the immense gravitational fields of these large cosmic structures and is deflected. Occasionally, if a background galaxy lies at a small angular distance from the cluster center, the lensing effects are highly nonlinear, leading to the formation of giant arcs and multiple-image systems. This regime is often called strong lensing. However, even at large angular distances, the light feels the gravitational pull of the cluster. In this case, where the lensing distortion changes on scales much larger than the size of the sources, the shape of the distant galaxies is only weakly distorted. In this weak lensing regime, the lensing effects are described by means of an additional image ellipticity.

Every cluster produces a weak lensing signal, but strong lensing events are rare and are often observed only in the cores of the most massive clusters or in systems with enhanced shear fields. Hennawi et al. (2007) and Meneghetti et al. (2010a) illustrated with the help of numerical simulations how peculiar the population of strong lensing clusters is. Clusters forming in the context of cold dark matter (CDM) typically have oblate, triaxial dark matter halos (Frenk et al. 1988; Dubinski \& Carlberg 1991; Muñoz-Cuartas et al. 2011; Limousin et al. 2013; Lemze et al. 2012; Despali et al. 2013), and, among them, strong lenses tend to have their major axes preferentially oriented along the line of sight. Additionally, as described in Torri et al. (2004), the cluster's ability to produce strong lensing features is boosted by dynamical events such as mergers or, more generally, by substructures orbiting around their host halo and occasionally crossing the cluster cores in projection (Bayliss et al. 2014).

For these reasons, the selection of clusters based on their ability to produce strong lensing events is likely to generate a sample affected by biases. Because lensing is sensitive to the total mass projected onto the lens plane, the halo structural parameters inferred from the lensing analysis of clusters affected by an orientation bias will be biased as well. In particular, for clusters elongated along the line of sight, we expect to measure higher masses and concentrations (see, e.g., Oguri et al. 2009; Oguri \& Blandford 2009; Hennawi et al. 2007; Meneghetti et al. 2010a; Gralla et al. 2011), and the opposite is expected for clusters whose major axes are perpendicular to the line of sight.

To avoid these issues, a selection based on the cluster X-ray morphology is often advocated. The thermal X-ray emission by galaxy clusters originates in the intracluster medium (ICM), which is ionized gas heated to temperatures up to $\sim 20 \mathrm{keV}$ emitting in the X-ray via thermal bremsstrahlung radiation (e.g., Sarazin 1986). In the absence of processes inducing nonthermal pressure contributions, for example perturbations induced by dynamical events like mergers or ICM turbulence, we do expect the ICM to be nearly in hydrostatic equilibrium with the cluster gravitational potential. As an indication for such equilibrium, or relaxation, the X-ray surface brightness is expected to be symmetric and its isocontours "round" and concentric (see, e.g., Rasia et al. 2013b). Following this philosophy, a Cluster Lensing and Supernova Survey with Hubble (CLASH) cluster sample (Postman et al. 2012) has been constructed by selecting 20 massive clusters from X-ray-based compilations of massive relaxed clusters. The relaxation state has been established on the basis of X-ray morphological estimators applied to Chandra $X$-ray Observatory images.

Are these selection criteria really leading to a sample that is unbiased in terms of lensing masses and concentrations? Giocoli et al. (2012a) have recently pointed out that for randomly selected cluster samples, the concentration-mass relation derived from a two-dimensional (2D) lensing analysis is expected to have a lower amplitude compared to the intrinsic three-dimensional (3D) concentration-mass relation. The reason is identified in the prolate triaxial shape of the cluster halos. Because of their prolateness, the probability of observing them elongated on the plane of the sky is higher than the probability of viewing them with their major axes pointing toward the observer (some examples are shown in Figure 10 of Gao et al. 2012). Rasia et al. (2013a) showed that selecting clusters according to their X-ray luminosity not only increases the normalization of the $c-M$ relation with respect to a control sample but also returns a steeper slope. This behavior is explained by the fact that at fixed mass, the most luminous clusters are also the most concentrated.

In this paper, we aim to use a set of numerical simulations of galaxy clustersized halos, the MUSIC-2 simulation set, to better understand the expected properties of a sample of clusters having $\mathrm{X}$-ray morphologies similar to the CLASH sample. In particular, we wish to quantify the possible residual biases on the mass and on the concentration estimates that are due to the CLASH selection function. This work has two companion papers ${ }^{34}$ : the strong lensing and weak shear study of CLASH clusters by Merten et al. (2014) and the weak lensing and magnification study of CLASH clusters by Umetsu et al. (2014), where a comparison between our results and the observational analysis of the CLASH sample is presented.

The paper is structured as follows. In Section 2, we introduce the simulation set used in our analysis, and we describe the methods used to measure the shape of density profiles in simulated halos. In Section 3, we introduce the CLASH cluster sample to which the simulations will be compared. In Section 4, we describe the morphological parameters used to construct a sample of X-ray-selected clusters resembling the properties of the CLASH clusters. In Section 5, we describe the general properties of the halos in the simulated set and discuss their concentration-mass relation. In Section 6, we discuss the concentration-mass relation of strong lensing and X-rayselected halos. In Section 7, we use the X-ray morphology of the simulated clusters to predict the concentrations of the individual CLASH clusters. Finally, Section 8 contains our summary and conclusions.

34 To appear on arXiv/astro-ph the same day as this work. 


\section{SIMULATIONS}

\subsection{The MUSIC-2 Sample}

The MUSIC-2 sample (Sembolini et al. 2013a, 2013b; Biffi et al. 2014) consists of a mass-limited sample of resimulated halos selected from the MultiDark cosmological simulation. This simulation is dark matter only and contains $2048^{3}$ (almost 9 billion) particles in a $\left(1 h^{-1} \mathrm{Gpc}\right)^{3}$ cube. It was performed in 2010 using ART (Kravtsov et al. 1997) at the NASA Ames Research Center. All of the data of this simulation are accessible from the online MultiDark database. ${ }^{35}$ The run was done using the best-fitting cosmological parameters to WMPA7+BAO+SNI $\left(\Omega_{M}=0.27, \Omega_{b}=0.0469, \Omega_{\Lambda}=0.73, \sigma_{8}=0.82, n=0.95\right.$, $h=0.7)$. This is the reference cosmological model used in the rest of the paper.

The halo sample was originally constructed by selecting all of the objects in the simulation box that are more massive than $10^{15} h^{-1} M_{\odot}$ at redshift $z=0$. In total, 282 objects were found above this mass limit. All of these massive clusters were resimulated both with and without radiative physics. The zooming technique described in Klypin et al. (2001) was used to produce the initial conditions for the resimulations. All particles within a sphere of $6 \mathrm{Mpc}$ radius around the center of each selected object at $z=0$ were found in a low-resolution version ( $256^{3}$ particles) of the MultiDark volume. This set of particles was then mapped back to the initial conditions to identify the Lagrangian region corresponding to a $6 h^{-1} \mathrm{Mpc}$ radius sphere centered at the cluster center of mass at $z=0$. The initial conditions of the original simulations were generated in a finer mesh of size $4096^{3}$. By doing so, the mass resolution of the resimulated objects was improved by a factor of eight with respect to the original simulations. The parallel TREEPM+SPH GADGET code (Springel 2005) was used to run all of the resimulations. We stress that during the resimulation process, we make sure that all of the clusters that have been included in the MUSIC database are free from contamination by lowresolution particles that are outside the Lagrangian region of the resimulated area. If an object is formed close to the boundary of the high-resolution region, it might be very likely affected by the presence of particles with different spatial and mass resolution. In this case, we exclude this object from our analysis because it is not properly simulated. All of the MUSIC objects used in the analysis thus have their Lagrangian regions well inside the high-resolution regions defined by the $6 h^{-1}$ Mpc spheres at $z=0$.

The MUSIC-2 sample exists in two flavors. In a first set of resimulations, baryons were added to the dark matter distributions extracted from the parent cosmological box, and their physics was simulated via smoothed particle hydrodynamics (SPH) techniques, without including radiative processes. A second set of resimulations accounts for the effects of radiative cooling, UV photoionization, star formation, and supernova feedback, including the effects of strong winds from supernovae.

In this paper, we focus our analysis on the nonradiative version of these simulations. Our choice is based on the fact that radiative simulations without a proper description of energy feedback from active galactic nuclei (AGNs) generally produce unrealistically dense cores because of the well-known overcooling problem (see, e.g., Borgani \& Kravtsov 2011). More recent simulations show that this problem is mitigated in simulations that simulate energy feedback from AGNs (Duffy

\footnotetext{
35 www.MultiDark.org
}

Table 1

Completeness Mass Limits and Number of Halos above the Completeness Mass Limits in the MUSIC-2 Sample

\begin{tabular}{lccc}
\hline \hline Redshift & $\begin{array}{c}\text { Mass Limit }\left(M_{\text {vir }}\right) \\
\left(h^{-1} M_{\odot}\right)\end{array}$ & $\begin{array}{c}\text { Mass Limit }\left(M_{200}\right) \\
\left(h^{-1} M_{\odot}\right)\end{array}$ & No. of Halos \\
\hline 0.250 & $6.3 \times 10^{14}$ & $4.3 \times 10^{14}$ & 128 \\
0.333 & $6.4 \times 10^{14}$ & $5.1 \times 10^{14}$ & 97 \\
0.429 & $6.0 \times 10^{14}$ & $5.0 \times 10^{14}$ & 80 \\
0.667 & $3.9 \times 10^{14}$ & $4.0 \times 10^{14}$ & 89 \\
\hline
\end{tabular}

et al. 2010; McCarthy et al. 2011; Planelles et al. 2014; Rasia et al. 2013a; Planelles et al. 2014). This physical ingredient is not yet included in the MUSIC-2 sample. Moreover, our intention is to correlate the profile measurements with the strong lensing efficiency of the simulated halos. Killedar et al. (2012), comparing simulations with different treatments of baryonic processes, find that the addition of gas in nonradiative simulations does not significantly change the strong lensing predictions. However, gas cooling and star formation together significantly increase the number of expected giant arcs and the Einstein radii by a nonrealistic amount, particularly for lower redshift clusters and lower source redshifts. Further inclusion of AGN feedback, however, reduces the predicted strong lensing efficiencies such that the lensing cross sections become closer to those obtained for simulations including only dark matter or nonradiative gas. The main requirements for this study are (1) a large number of highly resolved halos to accurately measure the profiles and determine the dependence of concentration on mass, and (2) the presence of gas in the simulations in order to allow their X-ray analysis (see Section 4). For these reasons, we choose to use the nonradiative version of the MUSIC-2 sample.

The mass resolution for these simulations corresponds to $m_{\mathrm{DM}}=9.01 \times 10^{8} h^{-1} M_{\odot}$ and to $m_{\mathrm{SPH}}=1.9 \times 10^{8} h^{-1} M_{\odot}$. The gravitational softening was set to $6 h^{-1} \mathrm{kpc}$ for the SPH and dark-matter particles in the high-resolution areas. Several lowmass clusters have been found close to the large ones and not overlapping with them. Thus, the total number of resimulated objects is considerably larger than originally identified in the parent cosmological box. In total, there are 535 clusters with $M$ $>10^{14} h^{-1} M_{\odot}$ at $z=0$ and more than 2000 group-like objects with masses in the range $10^{13} h^{-1} M_{\odot}<M_{\text {vir }}<10^{14} h^{-1} M_{\odot}$. In this study, we use a subsample of these halos, as explained below.

We have stored snapshots for 15 different redshifts in the range $0 \leqslant z \leqslant 9$ for each resimulated object. The snapshots that overlap with the redshifts of the CLASH clusters are at $z=0.250,0.333,0.429$, and 0.667 .

The sample is complete above the mass thresholds given in Table 1. To extend our analysis toward smaller masses and to be able to constrain the concentration-mass relation over a wider mass range, we also analyze halos with masses below the completeness limits. In particular, we use all halos with mass $M_{\text {vir }}>2 \times 10^{14} h^{-1} M_{\odot}$. Therefore, we investigate a total of 1,419 halos, summing all halos at different redshifts.

\subsection{Density Profiles}

\subsubsection{Generalities}

Navarro et al. (1996) argued that the density profiles of numerically simulated dark matter halos can be well fitted by an appropriate scaling of a "universal" function over a wide range of masses. The function suggested to fit these profiles was 
later dubbed the Navarro-Frenk-White density profile (NFW hereafter) and is given by

$$
\rho(r)=\frac{\rho_{s}}{\left(r / r_{s}\right)\left(1+r / r_{s}\right)^{2}},
$$

where $\rho_{s}$ and $r_{s}$ are the characteristic density and the scale radius of the halo. The profile is characterized by a logarithmic slope that is shallower than isothermal for $r \ll r_{s}$ and steeper than isothermal for $r \gg r_{s}$.

Subsequent numerical studies (see e.g., Navarro et al. 1997) confirmed that the NFW function is appropriate to describe the profiles of equilibrium halos, i.e., of systems that are close to being in virial equilibrium, and is now widely used to characterize the shape of cluster-sized halos both in observations and in simulations.

Along with the definition of the NFW density profile came that of the halo concentration, $c_{\Delta}=r_{\Delta} / r_{s}$, which is the ratio of the size of the halo, here defined as the radius enclosing a certain mean overdensity $\Delta$ above the critical density of the universe, $\rho_{\text {crit }}(z)$. The most appropriate value to describe the size of an equilibrium halo is its virial radius, i.e., the radius within which the halo particles are gravitationally bound and settled into equilibrium orbits. In this case the virial overdensity, $\Delta_{\text {vir }}$, is a function of cosmology and redshift (Bryan \& Norman 1998; Nakamura \& Suto 1997). To avoid this cosmological dependence, Navarro et al. (1996) adopted the round number of $\Delta=200$, which is commonly used in the literature independently of the assumed cosmological model. In this paper, we will also define the size of the halos as $r_{200}$, which is the radius enclosing a mean density $\bar{\rho}=200 \rho_{\text {crit }}(z)$. Diemer $\&$ Kravtsov (2014) recently showed that rescaling clusters to this radius returns a self-similar inner density profile.

Despite the fact that the profiles of equilibrium halos are well described by the NFW function, a large fraction of halos formed in a cosmological box are far from having reached virial equilibrium (Ludlow et al. 2012; Meneghetti \& Rasia 2013). Balmès et al. (2014) discussed the dependence of this fraction on cosmology, finding that it is particularly sensitive to dark energy. The reason is simply understood: dark energy affects the formation and the growth of the cosmic structures. In the case of nonequilibrium halos, the NFW function gives a poorer description of the shape of the density profiles, and other functions involving a larger flexibility (i.e., additional free parameters) may yield a preferable result. One example is the generalized NFW profile (gNFW; Zhao 1996), which is given by

$$
\rho(r)=\frac{\rho_{s}}{\left(r / r_{s}\right)^{\beta}\left(1+r / r_{s}\right)^{3-\beta}} .
$$

Compared to the NFW model, this profile is characterized by an additional parameter, namely the logarithmic inner slope $\beta$,

$$
-\frac{d \ln \rho}{d \ln r}=\beta
$$

which is radius-independent.

A strong debate exists in the literature about the inner slope of the density profile of simulated halos (see e.g., Moore et al. 1998; Newman et al. 2011). The advent of modern supercomputers allows us to push the mass and the spatial resolution of numerical simulations to unprecedented limits, and the new results indicate that there is a systematic deviation of the dark matter halo profiles from the form proposed by NFW (Merritt et al. 2006; Navarro et al. 2010). The function that best fits such profiles is the Einasto function (Einasto \& Haud 1989; Retana-Montenegro et al. 2012),

$$
\rho(r)=\rho_{-2} \exp \left\{-2 n\left[\left(\frac{r}{r_{-2}}\right)^{1 / n}-1\right]\right\},
$$

which is characterized by a running logarithmic slope,

$$
-\frac{d \ln \rho}{d \ln r} \propto r^{1 / n},
$$

parameterized in terms of the index $n$. The amplitude of the profile is set by the density $\rho_{-2}$, which is the density at the radius $r_{-2}$, i.e., at the radius where the logarithmic slope of the density profile is -2 .

\subsubsection{The Density Profiles of the MUSIC-2 Halos}

To describe the structural properties of the MUSIC-2 halos, we perform an analysis of their three-dimensional density profiles based on the functional forms introduced in this section. This analysis is done by fitting the Equations (1), (2), and (4) to the azimuthally averaged density profiles of the simulated halos. The code used to perform this analysis is the same used in another CLASH paper by Merten et al. (2014). ${ }^{36}$ As is common practice in the literature (e.g., Ludlow et al. 2013), we minimize the function

$$
R_{3 D}^{2}=\frac{1}{N_{\text {dof }}} \sum_{i}\left[\log _{10} \rho_{i}-\log _{10} \rho\left(r_{i}, \boldsymbol{p}\right)\right]^{2},
$$

where $\rho_{i}$ is the density measured in the $i$ th radial shell and $\boldsymbol{p}$ is the vector of parameters that are adjusted to derive the best-fitting function $\rho(r)$. In the case of the NFW profile, $\boldsymbol{p}=\left[\rho_{s}, r_{s}\right]$, and in the cases of the gNFW or Einasto profiles, $\boldsymbol{p}=\left[\rho_{s}, r_{s}, \beta\right]$ and $\boldsymbol{p}=\left[\rho_{s}, r_{s}, n\right]$, respectively. The variable $N_{\text {dof }}$ is the number of degrees of freedom, i.e., the number of radii at which the profiles are evaluated minus the number of free parameters in the fit.

When analyzing these three-dimensional density profiles, we perform the fit over the radial range $\left[\tilde{r}_{\min }, \tilde{r}_{200}\right]$, where $\tilde{r}_{\min }=0.02 R_{\mathrm{vir}}$, and $\tilde{r}_{200}$ is the true $r_{200}$ of the halo. Of course, the choice of the radial range over which the fit is performed is important because substructures located within this range can affect the result of the fit (Meneghetti \& Rasia 2013).

A similar analysis is performed on the two-dimensional profiles, i.e., on the azimuthally averaged surface-density profile, $\Sigma_{i}$, corresponding to an arbitrary line of sight to the halo. The details of this analysis are discussed in the paper by J. Vega et al. (in preparation). In this case, the fitting functions are the projections of the functions in Equations (1), (2), and (4):

$$
\Sigma(R)=2 \int_{0}^{r_{t}} \rho\left(r=\sqrt{R^{2}+\xi^{2}}\right) d \xi,
$$

where $\xi$ indicates the spatial coordinate along the line of sight, and $R$ is the projected radius. In the formula above, $r_{t}$ is a truncation radius, which is introduced to take into account that our halos are at the center of a cube with side length $r_{t}=6 h^{-1} \mathrm{Mpc}$ comoving. The figure-of-merit function to be minimized in this case is

$$
R_{2 D}^{2}=\frac{1}{N_{\mathrm{dof}}} \sum_{i}\left[\log _{10} \Sigma_{i}-\log _{10} \Sigma\left(R_{i}, \boldsymbol{p}\right)\right]^{2} .
$$

\footnotetext{
36 Based on the open-source library Levmar http://users.ics.forth.gr/lourakis/levmar/.
} 
In order to be consistent with the analysis done on the CLASH clusters, we perform the two-dimensional fits over the radial range $\left[20 h^{-1} \mathrm{kpc}, R_{\text {vir }}\right]$.

When projecting the cubes within which the halo particles are distributed, we expect that in particular for the smallest systems, there will be a two-halo contribution, which is not properly taken into account in the fitting procedure. To estimate if this may bias our conclusions, we repeat the fit using only the particles inside spheres with radius $R_{\text {vir }}$. The average concentrations do not change significantly, even at low masses, so we conclude that the two-halo contribution is a minor perturbation relative to the one-halo term for the radial scales we are probing $\left(R_{2 D}<R_{\text {vir }}\right)$.

For both the three- and the two-dimensional analyses, the best-fit parameters are used to compute the masses and the concentrations of the simulated halos. In the following, we identify the quantities estimated from these two analyses with the labels 3D and 2D, respectively. The best-fit masses are obviously obtained by integrating the best-fit density profiles,

$$
M=4 \pi \int_{0}^{r_{200}} \rho\left(r, \boldsymbol{p}_{\text {best }}\right) r^{2} d r .
$$

The value of $r_{200}$ used here is derived by solving the equation

$$
\frac{\int_{0}^{r_{200}} \rho\left(r, \boldsymbol{p}_{\text {best }}\right) r^{2} d r}{r_{200}^{3}}=\frac{200}{3} \times \rho_{\text {crit }}(z) .
$$

Using its original definition (NFW), the concentration is the ratio between $r_{200}$ and the scale radius, $r_{s}$. For the NFW profile, the scale radius corresponds to the radius where

$$
-\frac{d \ln \rho}{d \ln r}=2
$$

that is, where the density profile has an isothermal slope. In the rest of the paper, we adopt the same definition also for the gNFW and Einasto profiles,

$$
c_{200} \equiv \frac{r_{200}}{r_{-2}} .
$$

Note that for the gNFW the following relation holds between $r_{-2}$, the scale radius $r_{s}$, and the inner slope $\beta$ :

$$
\begin{aligned}
& r_{-2}=(2-\beta) r_{s} . \\
& \text { 2.3. Lensing Analysis }
\end{aligned}
$$

\subsection{Lensing Analysis}

The lensing analysis of the MUSIC-2 halos is described in detail in Vega et al. (in preparation). For the purpose of this paper, we use their estimates of the Einstein radii over a large number of projections per cluster. We also use their convergence profiles, properly rescaled into surface-density profiles, and their mass and concentrations based on the fits of the surfacedensity profiles. The masses $M_{2 D}$ and the concentrations $c_{2 D}$ are equivalent to the values derived from a comprehensive lensing analysis of real observations. Hence, we compare $M_{2 D}$ and $c_{2 D}$ to Merten et al. (2014) and Umetsu et al. (2014).

For this work, we use our consolidated lensing simulation pipeline (see, e.g., Meneghetti et al. 2010a and references therein). Briefly, the following steps are involved.

1. We project the particles belonging to each individual halo along the desired line of sight on the lens plane.
2. Starting from the position of the virtual observer, we trace a bundle of light rays through a regular grid of $2048 \times 2048$ covering a region of $1.5 \times 1.5 h^{-1} \mathrm{Mpc}$ around the halo center on the lens plane.

3. Using our code RayShoot (Meneghetti et al. 2010b), we compute the deflection $\boldsymbol{\alpha}(\boldsymbol{x})$ at each light-ray position $\boldsymbol{x}$, accounting for the contributions from all particles on the lens plane.

4. The resulting deflection field is used to derive several relevant lensing quantities. In particular, we use the spatial derivatives of $\boldsymbol{\alpha}(\boldsymbol{x})$ to construct the convergence, $\kappa(\boldsymbol{x})$, and the shear, $\boldsymbol{\gamma}=\left(\gamma_{1}, \gamma_{2}\right)$, maps. These are defined as

$$
\begin{gathered}
\kappa(\boldsymbol{x})=\frac{1}{2}\left(\frac{\partial \alpha_{1}}{\partial x_{1}}+\frac{\partial \alpha_{2}}{\partial x_{2}}\right), \\
\gamma_{1}(\boldsymbol{x})=\frac{1}{2}\left(\frac{\partial \alpha_{1}}{\partial x_{1}}-\frac{\partial \alpha_{2}}{\partial x_{2}}\right), \\
\gamma_{2}(\boldsymbol{x})=\frac{\partial \alpha_{1}}{\partial x_{2}}=\frac{\partial \alpha_{2}}{\partial x_{1}} .
\end{gathered}
$$

5. The lens critical lines are defined as the curves along which the determinant of the lensing Jacobian is zero (e.g., Schneider et al. 1992):

$$
\operatorname{det} A=(1-\kappa-|\gamma|)(1-\kappa+|\gamma|)=0 .
$$

In particular, the tangential critical line is defined by the condition $(1-\kappa-|\gamma|)=0$, whereas the radial critical line corresponds to the line along which $(1-\kappa+|\gamma|)=0$. In the following sections, we will often use the term Einstein radius to refer to the size of the tangential critical line. As discussed in Meneghetti et al. (2013), there are several possible definitions for the Einstein radius. In this paper, we adopt the effective Einstein radius definition (see also Redlich et al. 2012),

$$
\theta_{E} \equiv \frac{1}{d_{\mathrm{L}}} \sqrt{\frac{S}{\pi}},
$$

where $S$ is the area enclosed by the tangential critical line and $d_{\mathrm{L}}$ is the angular diameter distance to the lens plane.

All of the lensing quantities are computed for a source redshift $z_{\mathrm{s}}=2$.

In order to increase the statistics and to take into account possible projection effects, J. Vega et al. (in preparation) study each halo under a large number of lines of sight. More precisely, they investigate 100 lines of sight for the halos above the mass completeness limits and 30 projections for those below the completeness limit. This implies that for each halo, we have a catalog containing at least 30 measurements of the Einstein radius, projected mass, and projected concentration.

\subsection{X-Ray Analysis}

We build a mock X-ray catalog by producing for each simulated cluster three Chandra event files corresponding to orthogonal projections aligned with the Cartesian axes of the simulation. Because of excessive computational demand, we cannot investigate all of the lines of sight considered in J. Vega et al. (in preparation). The images are created by the X-ray 
MAp Simulator (X-MAS; Gardini et al. 2004), in which we utilize the ancillary response function and redistribution matrix function proper of the ACIS-S3 detector (for a complementary X-ray analysis of the MUSIC-2 sample, we refer the reader to Biffi et al. 2014). The field of view (FOV) covers (16 arcmin $)^{2}$. For the cosmology and redshifts analyzed, the FOV size is equivalent to the following physical scales: $5.43 h^{-1} \mathrm{Mpc}$ at $z=0.250,6.57 h^{-1} \mathrm{Mpc}$ at $z=0.333,7.71 h^{-1} \mathrm{Mpc}$ at $z=0.429$, and $9.57 h^{-1} \mathrm{Mpc}$ at $z=0.667$. The spectral emission is generated by adopting the MEKAL model in which we fix the redshift to the simulation's value and the metallicity to a constant value equal to 0.3 times the solar metallicity as tabulated by Anders \& Grevesse (1989). Finally, the contribution of the galactic absorption is introduced through a WABS model with $N_{\mathrm{H}}=5 \times 10^{20} \mathrm{~cm}^{-2}$ (see e.g., Lemze et al. 2009). The exposure time is set to $100 \mathrm{ks}$, allowing a fair comparison with observations.

\section{THE CLASH CLUSTER SAMPLE}

The Cluster Lensing and Supernova Survey with Hubble (CLASH) is a Multi-Cycle Treasury program with the Hubble Space Telescope (HST). During HST cycles 18-20, 524 orbits were dedicated to observing 25 massive galaxy clusters. Among the goals of the program is to use the gravitational lensing properties of these objects to accurately constrain their mass distributions. In particular, one of the key objectives is to establish the degree of concentration of dark matter in the cluster cores, a key prediction of structure formation models. The survey is described in detail in Postman et al. (2012).

The targets of the CLASH program were selected to minimize the lensing-based selection that favors systems with overly dense cores. Specifically, 20 CLASH clusters are solely $\mathrm{X}$-ray selected. The X-ray-selected clusters are massive $(k T>5 \mathrm{keV})$ and, in most cases, they appear to have a regular X-ray morphology. Five additional clusters are included for their lensing strength. These clusters have large Einstein radii $\left(\theta_{E}>35^{\prime \prime}\right)$ and were included to optimize the likelihood of finding highly magnified high $-z(z>7)$ galaxies. Using galaxy clusters as gravitational telescopes is another of the key objectives of CLASH, and the program has provided an extraordinary contribution to this field of research (Zheng et al. 2012; Bouwens et al. 2014; Bradley et al. 2014; Smit et al. 2013; Coe et al. 2013).

For each CLASH cluster, a large number of lensing constraints were collected, either from the HST, Subaru (e.g., Medezinski et al. 2013), or ESO/WFI (Gruen et al. 2013) telescopes or from the CLASH-VLT spectroscopic program (Balestra et al. 2013). Using these data of unprecedented quality, mass models for several CLASH targets have been published over the last few years employing different methods of reconstruction (Zitrin et al. 2011, 2012a, 2012b, 2013; Umetsu et al. 2012; Coe et al. 2012; Medezinski et al. 2013). These techniques are based on strong, weak, or a combination of strong and weak lensing.

In two companion papers, Merten et al. (2014) and Umetsu et al. (2014) focus on the analysis of the X-ray-selected subsample of CLASH clusters. In Merten et al. (2014), a well-tested reconstruction method (Merten et al. 2009, 2011; Meneghetti et al. 2010b; Rasia et al. 2012) is used to combine weak and strong lensing constraints and derive the convergence maps of these clusters. Fitting the surface-density profiles extracted from the maps, they measure the masses and concentrations of the CLASH clusters. As stated, the X-ray-selected CLASH clus- ters are ideal for this density profile analysis. In this paper, we analyze the MUSIC-2 halo sample with the intent of deriving theoretical expectations to compare to the results of the observational analysis of Merten et al. (2014) and Umetsu et al. (2014).

\section{X-RAY SELECTION}

\subsection{X-Ray Morphological Parameters}

One of the goals of this paper is to identify halos in the MUSIC-2 sample that closely resemble the X-ray properties of the clusters in the CLASH X-ray-selected sample. Because this sample was selected to have a high degree of regularity in the $\mathrm{X}$-ray morphology, we try to find equivalents in the simulations that mimic these X-ray characteristics.

We use five parameters to measure the X-ray morphology in the soft-energy band ([0.5-2] keV) images of our halos. These morphological parameters are evaluated within a physical radius $R_{\max }=500 \mathrm{kpc}$ following the same procedure adopted in the $\mathrm{X}$-ray analysis of the CLASH clusters. The results of this analysis will be presented in detail in a forthcoming paper by Donahue et al. (2014). The five parameters are:

1. the centroid-shift, $w$, which assesses how much the centroid of the X-ray surface brightness moves when the aperture radius used to compute it decreases from $R_{\max }$ to smaller values. It is defined as

$$
w=\frac{1}{R_{\max }} \times \sqrt{\frac{\Sigma\left(\Delta_{i}-\langle\Delta\rangle\right)^{2}}{N-1}},
$$

where $N$ is the total number of apertures considered and $\Delta_{i}$ is the separation of the centroids computed within $R_{\max }$ and within the $i$ th aperture;

2. the ellipticity, $e=1-b / a$, where the axial ratio is equal to the ratio of the square root of the eigenvalues obtained by diagonalizing the inertia tensor of the X-ray surface brightness evaluated within $R_{\max }$ (Buote \& Canizares 1992);

3. the X-ray-brightness concentration, which is the ratio between the integral of the surface brightness $S$ within two apertures with radii $100 \mathrm{kpc}$ and $R_{\max }$,

$$
c_{X}=\frac{S(r<100 \mathrm{kpc})}{S\left(r<R_{\max }\right)}
$$

Cassano et al. (2010);

4. and 5. the third- and fourth-order power ratios, $P_{3}$ and $P_{4}$. These are the third- and fourth-order multipoles of the surface-brightness distribution within an aperture of radius $R_{\text {ap }}=R_{\max }$. The generic $m$-order power ratio $(m>0)$ is defined as $P_{m} / P_{0}$ with

$$
P_{m}=\frac{1}{2 m^{2} R_{\mathrm{ap}}^{2 m}}\left(a_{m}^{2}+b_{m}^{2}\right) \quad \text { and } \quad P_{0}=a_{0} \ln \left(R_{\mathrm{ap}}\right),
$$

where $a_{0}$ is the total intensity within the aperture radius Buote \& Tsai (1996). The generic moments $a_{m}$ and $b_{m}$ are expressed in polar coordinates, $R^{\prime}$ and $\phi^{\prime}$, and given by

$$
a_{m}(r)=\int_{R^{\prime} \leqslant R_{\mathrm{ap}}} S\left(x^{\prime}\right) R^{\prime} \cos \left(m \phi^{\prime}\right) d^{2} x^{\prime},
$$

and

$$
b_{m}(r)=\int_{R^{\prime} \leqslant R_{\mathrm{ap}}} S\left(x^{\prime}\right) R^{\prime} \sin \left(m \phi^{\prime}\right) d^{2} x^{\prime} .
$$


For a review of X-ray morphological parameters, we refer to Rasia et al. (2013b).

The five morphological parameters introduced above are combined to define a global degree of X-ray regularity. Such a quantity is measured with respect to the mean of the simulated sample. Note that with reference to the X-ray appearance, we use the term "regular" to indicate halos with unperturbed surface brightness distributions (Rasia et al. 2012). Very often, these halos are called "relaxed." We do not use this term to differentiate from the classification discussed in Section 5.2. Regular halos have small centroid shift, ellipticity, and power ratios. In addition, they have large surface-brightness concentrations. Thus, we define the regularity parameter

$$
\begin{aligned}
M= & \left(\frac{\log _{10}(w)-\left\langle\log _{10}(w)\right\rangle}{\sigma_{\log _{10} w}}\right)+\left(\frac{e-\langle e\rangle}{\sigma_{e}}\right) \\
& +\left(\frac{\log _{10}\left(1 / c_{X}\right)-\left\langle\log _{10}\left(1 / c_{X}\right)\right\rangle}{\sigma_{\log _{10} 1 / c_{X}}}\right) \\
& +\left(\frac{\log _{10}\left(P_{3}\right)-\left\langle\log _{10}\left(P_{3}\right)\right\rangle}{\sigma_{\log _{10} P_{3}}}\right) \\
& +\left(\frac{\log _{10}\left(P_{4}\right)-\left\langle\log _{10}\left(P_{4}\right)\right\rangle}{\sigma_{\log _{10} P_{4}}}\right)
\end{aligned}
$$

similarly to the $M$ parameter derived in Rasia et al. (2013b). In the formula above, each morphological parameter, $p_{i}$, is compared to its mean over the simulated halos, $\left\langle p_{i}\right\rangle$, and rescaled by the standard deviation of its distribution, $\sigma_{p_{i}}$.

By plugging the parameters $p_{\mathrm{CLASH}, i}$ measured on the X-ray images of the CLASH clusters into Equation (24), we use the $M$ parameter to quantify the regularity of the CLASH clusters with respect to the simulations. The $M$ parameters of the CLASH $\mathrm{X}$-ray-selected clusters are listed in Table 3. To construct a sample of CLASH-like clusters, we select the simulated halos having a regularity parameter similar to the observed clusters.

For the purpose of matching simulated halos to each individual CLASH cluster, we define the parameter $C_{X}$, which is defined as the distance, in parameter space, between each CLASH cluster and the simulated halos:

$$
C_{X}=\sum_{i=1,5}\left(\frac{p_{i}-p_{\mathrm{CLASH}, i}}{\sigma_{p_{i}}}\right)^{2},
$$

where $p_{i}=\left[\log _{10}(w), e,-\log _{10}\left(c_{X}\right), \log _{10} P_{3}, \log _{10} P_{4}\right]$ are the morphological parameters discussed above and $\sigma_{p_{i}}$ their standard deviations. As a result, the sample constructed via the $M$ parameter has an X-ray regularity similar to the CLASH sample. When we match halos using $C_{X}$, we identify only the simulated halos closest to each individual CLASH cluster in the morphological parameter space.

\subsection{Nonradiative versus Radiative Simulations}

While our choice to use the nonradiative version of the MUSIC-2 halos is motivated by the need to avoid biases caused by overcooling, it is well known that hydrodynamical simulations like those employed here poorly describe several X-ray properties of real clusters (Borgani \& Kravtsov 2011; Kravtsov \& Borgani 2012). For this reason, we do not use gas temperatures or X-ray luminosities to match the CLASH clusters in our simulations. Our comparison is based solely on the X-ray morphology.
To evaluate how the morphological parameters used in this work are influenced by the treatment of the gas, we use the hydrodynamical simulations described in Fabjan et al. (2010) and in Bonafede et al. (2011; see also Killedar et al. 2012; Planelles et al. 2014). These simulations, performed in the framework of a cosmological setting similar to that of the MUSIC- 2 simulations, exist both in nonradiative and radiative versions. Contrary to the MUSIC-2 simulations, the effects of AGN feedback are also included in the radiative case. The sample is significantly smaller, though. Seventy of these halos were recently processed with the X-MAS simulator, both in the nonradiative and radiative versions. We use this analysis to quantify the impact of radiative processes on the morphological parameters.

The distributions derived from the two simulated sets are consistent for all morphological parameters computed within $500 \mathrm{kpc}$, with the exception of the light concentration, which is lower in the radiative simulation because part of the central gas is turned into star and contributes less to the X-ray central emission. Applying the selection method based on the parameter $C_{X}$ on the halos in these two data sets for a few CLASH clusters, we obtained identical matches. Therefore, we can assume that our X-ray selection method can safely be used on the nonradiative simulations.

Notice that the two samples are characterized by similar concentration distributions, as shown in Rasia et al. (2013a). In particular, that paper found the following results: (1) the $\mathrm{c}-\mathrm{M}$ relations have similar slopes independent of the physics, and (2) the normalization of the $\mathrm{c}-\mathrm{M}$ relation in radiative simulations with no AGN feedback is $\sim 20 \%$ higher than that of the nonradiative simulations; the c-M relation from simulations including AGN feedback has a normalization similar to that of the nonradiative simulations.

\subsection{Example of a Regular Cluster: $A 383$}

To illustrate how our selection based on the X-ray morphology performs, we discuss the case of A383 (Allen et al. 2008), which is the first cluster observed in the framework of the CLASH program. A383 is a galaxy cluster at redshift $z=0.189$ (see, e.g., Zitrin et al. 2011). In the X-ray, it exhibits a very regular morphology, with nearly circular surface brightness contours (ellipticity 0.04; Postman et al. 2012). An X-ray image taken from the Archive of Chandra Cluster Entropy Profile Tables (ACCEPT) is shown in the small inset at the center of Figure 1. The image subtends $\sim 3.45$.

The four largest panels of Figure 1 show a sequence of simulated Chandra observations of MUSIC-2 halos corresponding to increasing values of $C_{X}$, which are annotated on the images. The top left panel shows the X-ray morphology of the halo that best matches A383 $\left(C_{X}=0.2\right)$. The X-ray morphology is indeed very similar to that of the observed cluster. As $C_{X}$ increases, the differences between the simulated and the true X-ray morphologies become more significant. On the basis of this and other visual inspections, we verified that $C_{X} \sim 0.4$ represents a good limit to select the halos "similar" to the true cluster.

\subsection{Example of Disturbed Cluster: MACSJ 1149}

Our selection successfully identifies simulated halos that also closely resemble more perturbed clusters. For example, this is the case for MACSJ 1149 (Ebeling et al. 2007), which is one of the CLASH clusters identified as high-magnification clusters, i.e., not included in the X-ray-selected sample. A comparison 


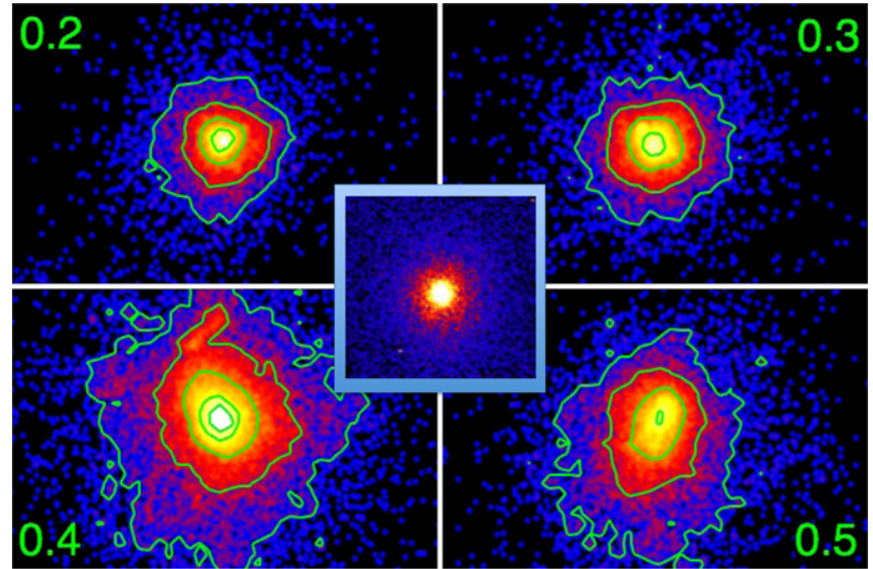

Figure 1. Examples of simulated clusters that match the CLASH cluster A383 (shown in the small inset) with four increasing values of $C_{X}$.

(A color version of this figure is available in the online journal.)

between the true X-ray morphology and that of a simulated halo with $C_{X}=0.18$ is shown in Figure 2, where we show the true Chandra image of the cluster in the smaller inset on the right.

Clearly, the degree of asymmetry and of elongation of the surface-brightness distribution in the simulated observation matches very closely that of MACSJ 1149.

\section{RESULTS}

In this section, we discuss the results of our analyses on cluster mass profiles. First, we focus on the intrinsic properties of the whole sample, i.e., we do not apply any selection method to match the properties of the CLASH clusters. We compare to existing studies in the literature to verify the consistency of our and previous results. Then we apply the selection based on the $\mathrm{X}$-ray selection and perform a one-to-one comparison between the simulated halos and each CLASH cluster.

\subsection{Relaxed and Unrelaxed Halos}

In this section we differentiate between relaxed and unrelaxed halos on the basis of a few criteria that are commonly used in the literature. Following the most restrictive approach proposed by Neto et al. (2007), we classify as strictly relaxed (or superrelaxed, as we dub them later in the paper) those objects satisfying the following properties.

1. Their center of mass displacement, defined as the offset between the center of mass (determined using all of the particles within the virial radius) and the minimum of the potential, in units of the virial radius, is $s=\left(\boldsymbol{r}_{\mathrm{cm}}-\right.$ $\left.\boldsymbol{r}_{\phi}\right) / r_{\text {vir }}<0.07$.

2. Their virial ratio is $\eta=2 T /|U|<1.35$, where $T$ is the kinetic energy and $U$ is the gravitational energy, computed using the particles within the virial radius.

3. Their substructure mass fraction, computed as the mass in resolved substructures within the virial radius, is $f_{\text {sub }}<0.1$.

Applying these selection criteria to the MUSIC-2 halos results in a fraction of relaxed halos of about $14.9 \%$ at redshift $z=0.25$. The fraction is reduced to $11.7 \%$ at redshift 0.333 , and it further drops to $10.4 \%$ and $8.9 \%$ at redshifts 0.429 and 0.667 , respectively.

Other authors use less restrictive or alternative criteria to identify the relaxed systems (e.g., Skibba \& Macciò 2011;

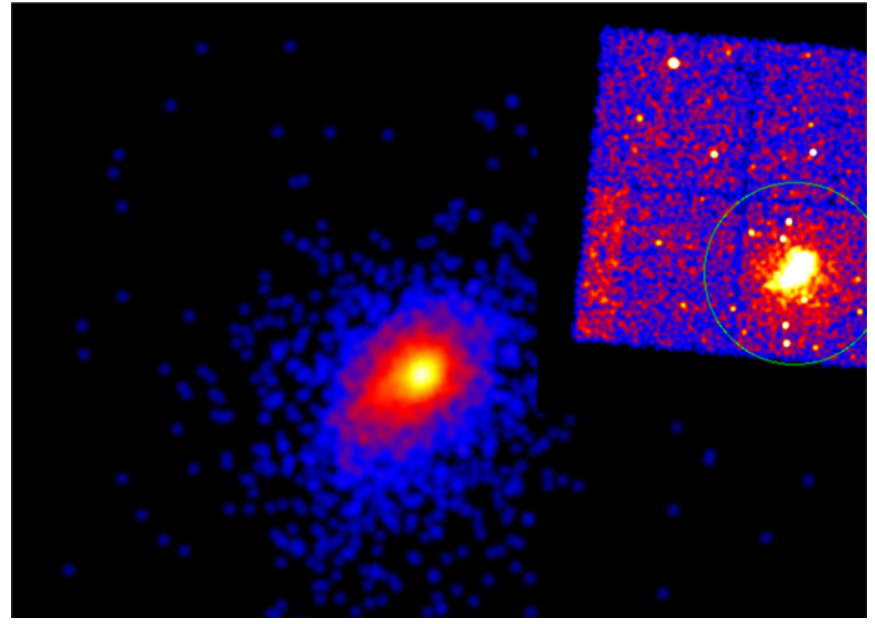

Figure 2. Best match to the morphologically disturbed cluster MACSJ 1149. The real X-ray image of the cluster is shown in the small inset on the right.

(A color version of this figure is available in the online journal.)

Skibba et al. 2011). For example, Bhattacharya et al. (2013) only use the center-of-mass displacement. In their paper, they report that the addition of the two other conditions on $\eta$ and $f_{\text {sub }}$ does not significantly affect the selection. On the contrary, we find that using only the center-of-mass displacement we end up with a significantly higher fraction of halos being classified as relaxed. This fraction amounts to $\sim 60 \%$ at $z=0.250$ and decreases to $\sim 51 \%$ at $z=0.667$. Such fractions are compatible with those quoted by Bhattacharya et al. (2013) (see also Biffi et al. 2014). Sembolini et al. (2013a) recently used the center-of-mass displacement in combination with the virial ratio to identify relaxed systems in simulations. They report that the relation between $\eta$ and $s$ becomes flat for $s \lesssim 0.1$, thus indicating that $\eta$ does not impact severely on the selection of relaxed systems. For our sample, the combination of $s$ and $\eta$ yields a fraction of relaxed halos corresponding to $47 \%$ at $z=0.250$, which decreases to $29 \%$ at $z=0.667$.

In the following sections, we will study the properties of the MUSIC-2 halos, dividing them into three subsamples. First, we will consider all halos, regardless of their relaxation state. Second, we will set the limit defined above on the center-ofmass displacement to construct the subsample of relaxed halos. Third, we will further downsize the sample by using all three criteria described above to identify the super-relaxed halos.

\subsection{Density Profiles}

As explained in Section 2.2, we fit the density profiles of the MUSIC-2 halos using the functions in Equations (1), (2), and (4). In Figure 3, we show the results of the fitting procedure. We quantify the goodness of fit by means of the residuals given in Equations (6) and (8).

The upper left panel shows the distributions of the fit residuals for the entire MUSIC-2 sample. When all halos are considered, regardless of their relaxation state, the NFW profile is the worstfitting model, i.e., the one with the largest residuals (see also Meneghetti \& Rasia 2013). This is not surprising given that the NFW model has one free parameter less than the gNFW or the Einasto profiles. However, this result highlights the difficulty of fitting all profiles with a universal law. Because the gNFW and the Einasto functions generally provide better fits to the profiles, we may use the statistical distributions of their residuals to identify the halos deviating significantly from the NFW form. 

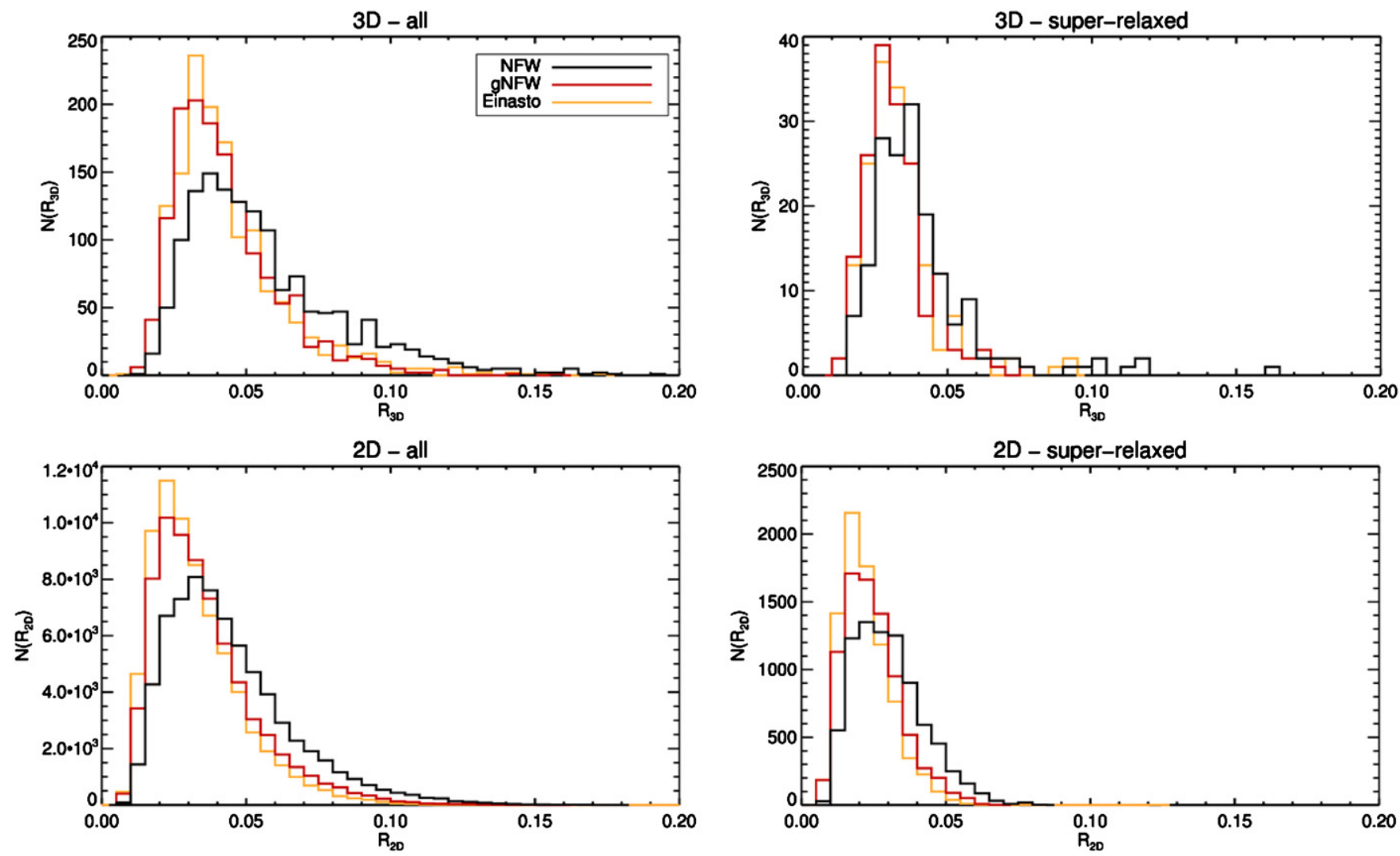

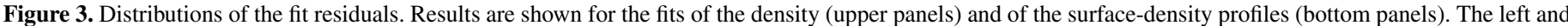

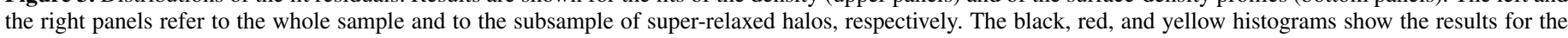
NFW, gNFW, and Einasto models.

(A color version of this figure is available in the online journal.)

As can be seen from Figure 3, such distributions are nearly log-normal, which suggests that halos having too-large NFW residuals compared to the Einasto and gNFW models may be identified via their deviation $\delta=\ln R_{3 D, \mathrm{NFW}}-\left\langle\ln R_{3 D, x}\right\rangle$, where $\left\langle\ln R_{3 D, x}\right\rangle$ is the mean value of $\ln R_{3 D}$ for either the Einasto or the gNFW model. Using this criterion, we find that about $40 \%$ of the halos have NFW fits resulting in too-large residuals compared to what is typically found by fitting with more flexible profiles.

This fraction drops to $\sim 19 \%$ and $\sim 6 \%$ if only relaxed and super-relaxed halos are considered. The distributions of the fit residuals for the super-relaxed subsample are shown in the upper right panel of Figure 3. For these halos, the NFW model is only a slightly worse fit compared to the gNFW and Einasto models.

In Figure 4 we see that the profiles that most deviate from the NFW form have inner slopes $\beta$ (resulting from the gNFW fits) that significantly differ from unity: their profiles are steeper or shallower than the NFW model. There is a slight indication for preferring a steep over a shallow slope (see also Figure 5). Indeed, the mean value of the inner slope $\beta$, measured for the whole sample, is $\langle\beta\rangle=1.03 \pm 0.31$, where the error is the rms in the sample. We also find that the goodness of the gNFW fit is not correlated with the inner slope $\beta$, i.e., shallow or steep inner slopes are not systematically the result of a bad gNFW fit.

When fitting the surface-density profiles, we find again that the NFW model is generally the worst-fitting function among the three models employed in this work. This is shown in the bottom left panel of Figure 3. Again, we find that restricting the analysis to the relaxed halos reduces the differences between the residual

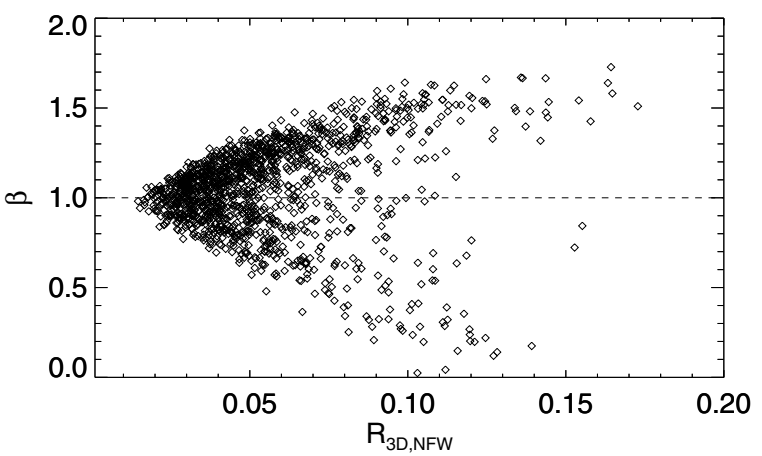

Figure 4. Inner slopes as they result from fitting the halo density profiles with gNFW models vs. the residuals of the NFW fits.

distributions of the NFW and gNFW or Einasto fits. However, from the results shown in the bottom right panel of Figure 3, it appears that a fraction of halos that are well fitted by NFW models in 3D are not NFW-like in projection. This result must be caused by the halo triaxiality and by the effects of substructures and additional matter along the line of sight. The work of $\mathrm{J}$. Vega et al. (in preparation), from which the 2D analysis shown here is taken, investigates the effects of triaxiality on the shape of the surface-density profiles of the CLASH clusters. We refer the reader to that paper for more details. We note that the halo surface-density profiles were derived by using all of the particles in a cylinder centered on the halo and with depth $6 h^{-1} \mathrm{Mpc}$. 


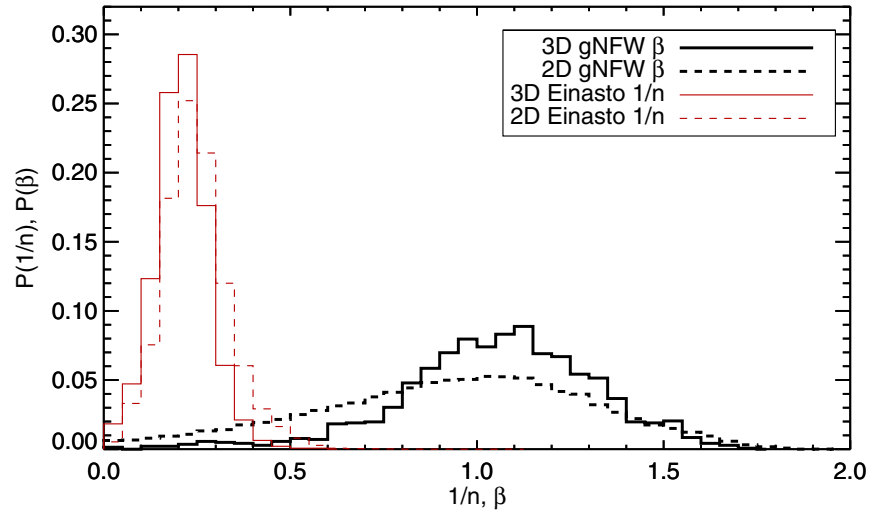

Figure 5. Distributions of the inner slopes obtained from the gNFW fits $(\beta)$ and of the Einasto index $1 / n$ derived from the analysis of the density (solid histograms) and of the surface-density profiles (dashed histograms) of the MUSIC-2 halos, as they result from fitting the halo density profiles with gNFW models vs. the residuals of the NFW fits.

(A color version of this figure is available in the online journal.)

The distributions of the inner slopes obtained from the gNFW fits of the surface-density profiles are shown in Figure 5 (thick histograms). We find that a large number of halos have rather flat profiles in 2D. The mean value of $\beta$ is $\langle\beta\rangle=0.89 \pm 0.47$. About $33 \%$ (15\%) of the halo projections are fitted with $\beta \leqslant 0.8$ $(\leqslant 0.5)$. The red histograms show the distributions of the Einasto indexes $1 / n$. The indexes obtained from the fit of the density profiles are slightly smaller than what are obtained from the fit of the surface-density profiles. The smaller the $1 / n$, the steeper the inner profile. The mean values are $\langle 1 / n\rangle=0.21 \pm 0.07$ and $\langle 1 / n\rangle=0.24 \pm 0.09$ for the $3 \mathrm{D}$ and $2 \mathrm{D}$ distributions, respectively. Such Einasto slopes appear to be in excellent agreement with the recent results of Dutton \& Macciò (2014).

To summarize, the halos in the MUSIC-2 sample span a wide range of structural parameters. As expected, the density profiles can differ significantly from the NFW form, and their shape can be better described with more flexible functions, such as the Einasto or gNFW models. When projecting the mass distributions, the scatter in the profile parameters and the deviation from the NFW model become even larger.

\subsection{Cluster Masses}

Having determined the level of diversity among density and surface-density profiles of the MUSIC-2 halos, we consider now how precisely the halo masses are derived from the profile fits. We consider both the cases of 2D and 3D masses, the former being the masses derived by deprojecting the best-fit models of the surface-density profiles under the assumption of spherical symmetry, and the latter those derived from the fits of the density profiles. Note that when measuring the $2 \mathrm{D}$ masses, we are not simulating any lensing analysis at this stage. In particular, we are not considering additional sources of systematics that may depend on the particular method to derive the mass from the weak and the strong lensing signals. Other works have shown that different methods of analysis may introduce systematic errors that are due, for example, to the presence of substructures inside and outside the clusters (Meneghetti et al. 2010b; Becker \& Kravtsov 2011; Rasia et al. 2012) and to the Bright Central Galaxy (Giocoli et al. 2013). Nevertheless, this exercise gives us important information on the intrinsic limits of the mass measurements based on the analyses of azimuthally averaged density or surface-density profiles.
We begin with the 3D masses. The distributions of the ratios between such masses and the true halo masses are shown in the left panel of Figure 6. The results are shown for the three fitting functions employed in this work (black, red, and orange histograms). We find that the masses recovered from the azimuthal fits of the density profiles are generally in good agreement with the true masses. The best agreement is obtained with the Einasto and gNFW profiles, with a slight preference for the first. These fits provide ratios around unity with $\mathrm{rms}=0.06$ and 0.05 , respectively. The masses estimated through the NFW fits are also in good agreement with the true masses. In this case, the median (mean) ratio is $0.98(0.97)$ and the distribution is twice as broad as in the two previous cases. The purple histogram is constructed by choosing, for each cluster, the mass estimate derived from the fitting function leading to the smallest residuals. In other words, we choose the most reliable mass estimate among those obtained with the three fitting functions. In most cases, the best model is the Einasto profile. Thus, the purple and the orange histograms are nearly coincident.

The histograms shown here refer to the whole halo sample, regardless of the relaxation state. As shown in the previous section, the density profiles of the relaxed halos are generally equally well fitted by NFW, gNFW, or Einasto models. Indeed, restricting the analysis to these halos, we find smaller rms for all three kinds of fit $(\lesssim 0.03)$, with mean and median ratios very close to unity. Despite the fact that the fraction of relaxed halos varies with redshift, we find that the mean mass ratios and their scatter remain constant as a function of redshift.

Even when fitting the surface-density profiles, the mass estimates $\left(M_{2 \mathrm{D}}\right)$ deviate only slightly from the true masses. The 2D masses appear to be underestimated by $\sim 5 \%$ on average, with the NFW and gNFW fits being slightly more biased than the Einasto fits. However, the scatter is much larger $(\sim 13 \%-14 \%)$ than for the 3D masses. The larger scatter is expected, given that the masses are derived under the assumption of spherical symmetry. Halos are generally triaxial, and projection effects can easily cause the mass to be over- or underestimated by a significant amount, depending on the halo orientation (see, e.g., Meneghetti et al. 2010b). As reported by Giocoli et al. (2012a), the halo prolateness may also cause a systematic underestimation of the mass derived from the 2D analysis. Assuming the triaxial model of Jing \& Suto (2002), they estimate this bias to be of order $\sim 10 \%$.

As in the left panel, the purple histogram in the right panel of Figure 6 shows the distribution of the ratios between the best 2D mass estimate and the true mass. Again, the distribution is close to that obtained by fitting with the Einasto profile.

On the basis of this result, we conclude that we should expect a modest negative bias of $\sim 5 \%$ on the mass estimates obtained by fitting the surface-density (or the convergence) profiles of galaxy clusters. This is due to the prolate shape of the halos, which are more frequently elongated on the plane of the sky than along the line of sight. The choice of the NFW or gNFW models to fit the halos tend to slightly increase the bias, and the opposite occurs with the Einasto profile.

If we repeat this analysis on the samples of relaxed and super-relaxed halos, we find that the mass bias tends to become smaller. In fact, the 2D masses deviate from the true masses by only $\sim 1 \%-2 \%$ in these cases. If the bias originates from halo triaxiality, this suggests that the most relaxed systems must generally be more spherical. In Figure 7, we show the distribution of the axis ratios $b / a$ and $c / a$ of all of the MUSIC-2 halos (color map). Here $a, b$, and $c$ are the semi-axes of the 

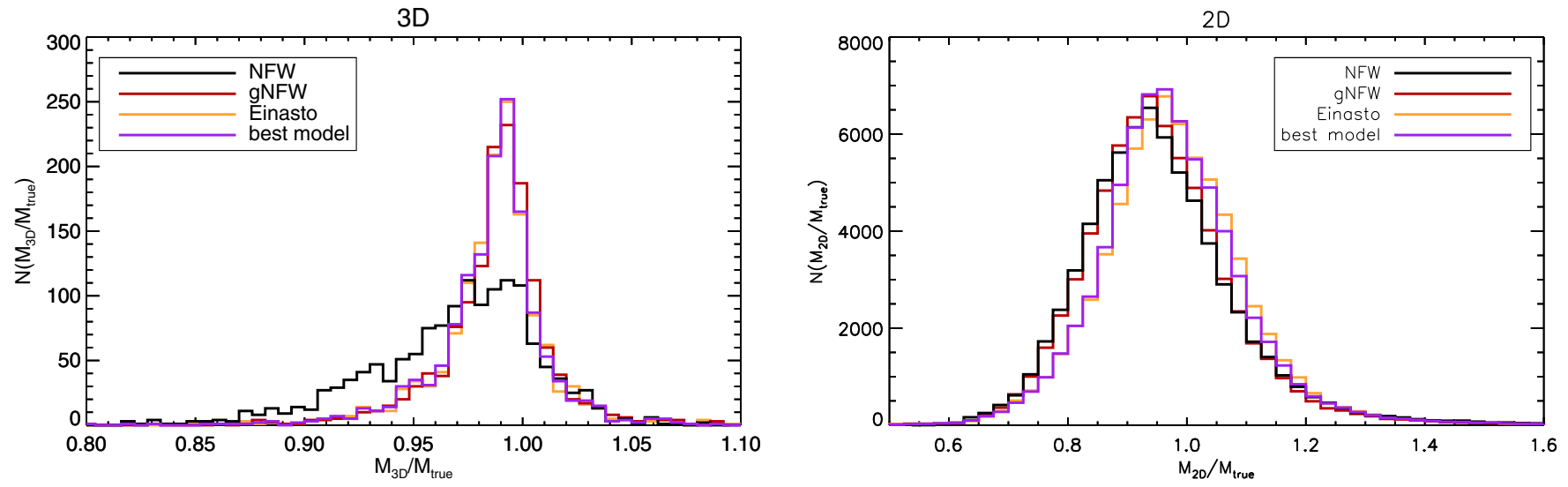

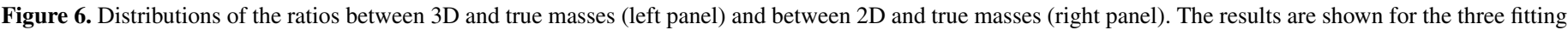

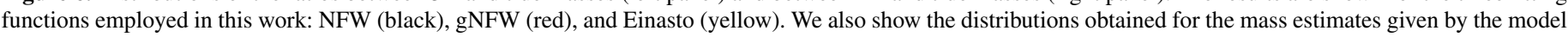
with the lowest residuals.

(A color version of this figure is available in the online journal.)

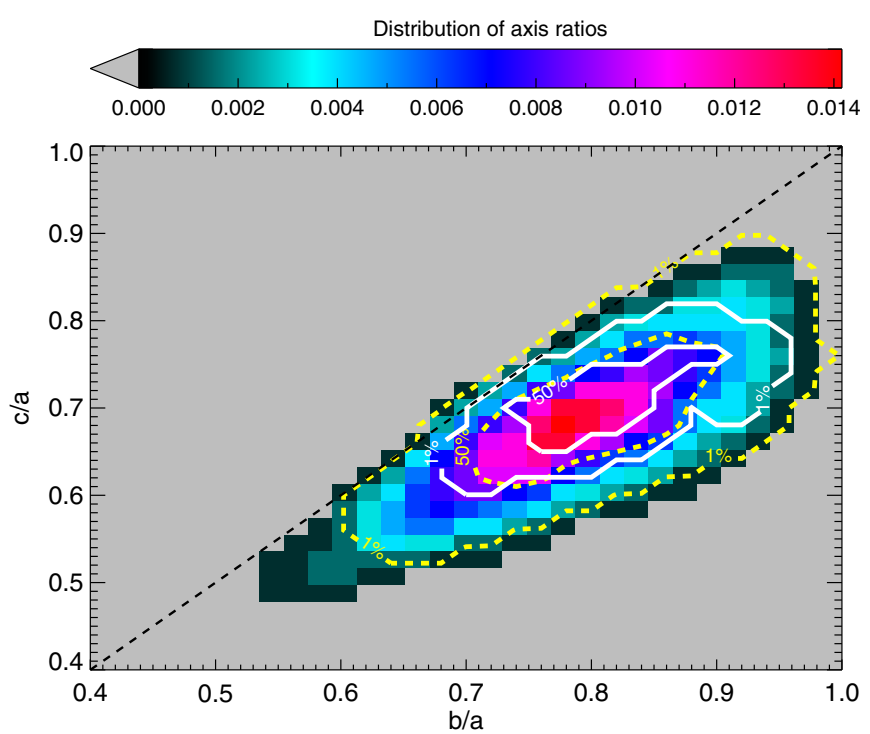

Figure 7. Color map shows the distribution of the axis ratios $b / a$ and $c / a$ of all of the MUSIC- 2 halos. The dashed and solid contours indicate the levels corresponding to $1 \%$ and $50 \%$ of the peaks of the distributions for the relaxed and super-relaxed halos.

(A color version of this figure is available in the online journal.)

inertial ellipsoid fitting the mass distribution of the halos with $a>b>c$. This fit is done using all particles within the virial radius. It is clear from this plot that the relaxed (yellow dashed contours) and super-relaxed systems (white contours) generally have higher values of both $b / a$ and $c / a$. Thus, their shape is closer to spherical than that of nonrelaxed halos, in agreement with Lemze et al. (2012).

\subsection{Concentration-mass Relation}

The concentration-mass $(c-M-z)$ relation is derived by means of nonlinear least-squares fitting using a Levenberg-Marquardt algorithm. The fitting function we employ is

$$
c(M, z)=A\left(\frac{1.34}{1+z}\right)^{B}\left(\frac{M}{8 \times 10^{14} h^{-1} M_{\odot}}\right)^{C}
$$

which was also used by Duffy et al. (2008) and De Boni et al. (2013), although using a different pivot mass and redshift. We perform this analysis for the three fitting models considered and report the corresponding best-fit parameters and errors in Table 2. The results are reported for the full sample as well as for the subsamples of relaxed and super-relaxed halos. We use Equation (26) to fit the $c-M-z$ relations derived from the analyses of the density profiles.

\subsubsection{Comparison between Fitting Models}

In the following, we consider the concentrations obtained from the NFW fit of the density profiles as a reference when making comparisons with the concentrations derived from the gNFW and Einasto fits. The yellow and green histograms in the upper panel of Figure 8 show the the distributions of the ratios $c_{3 \mathrm{D}, \mathrm{gNFW}} / c_{3 \mathrm{D}, \mathrm{NFW}}$ and $c_{3 \mathrm{D} \text {, Einasto }} / c_{3 \mathrm{D}, \mathrm{NFW}}$ obtained from our analysis. In both cases, we find that the distributions peak at values around $\sim 0.9-0.95$, with the Einasto concentrations being generally smaller than the NFW ones. This result is in agreement with the recent findings of Dutton \& Macciò (2014), who also find that the Einasto concentrations are $\sim 10-15 \%$ smaller than the NFW concentrations on the mass scale of the MUSIC-2 halos. The halos with the smallest concentrations are of course the unrelaxed systems, for which we already pointed out that the NFW model is generally a bad fit. An example of such profiles is shown in the bottom panel of Figure 8. In this case, the best-fit NFW concentration is $c_{3 \mathrm{D}, \mathrm{NFW}}=2.5$, and the gNFW and Einasto concentrations are $c_{3 \mathrm{D}, \mathrm{gNFW}}=10^{-2}$ and $c_{3 \mathrm{D} \text {,Einasto }}=0.1$, respectively. Considering only the relaxed or the super-relaxed halos, the ratios between fitted and true concentrations are much closer to unity. For example, the mean ratios of $c_{3 \mathrm{D}, \mathrm{gNFW}} / c_{3 \mathrm{D}, \mathrm{NFW}}$ and $c_{3 \mathrm{D} \text {, Einasto }} / c_{3 \mathrm{D}, \mathrm{NFW}}$ for the superrelaxed systems are 1.0 and 0.99 , respectively. We want to stress that the concentration of the Einasto profile being smaller than the NFW does not imply necessarily that the halos are less concentrated. For the Einasto profile, the mass inside the scale radius also depends on the $1 / n$ parameter. A halo with the same mass ratio between two radii as given by the NFW model can be fitted with a smaller concentration and a larger $n$.

In Figure 9, we show the $c-M$ relations obtained from fitting the density profiles of the MUSIC-2 with the NFW, gNFW, and Einasto models (upper, middle, and bottom panels, respectively). The results are displayed for the halos at the 
Table 2

Best-Fit Parameters for the 3D and 2D $c-M-z$ Relations

\begin{tabular}{|c|c|c|c|c|c|c|}
\hline Fitting Func. & $3 \mathrm{D} / 2 \mathrm{D}$ & Relax. & $A$ & $B$ & $C$ & Sel. Func \\
\hline NFW & $3 \mathrm{D}$ & all & $3.757 \pm 0.054$ & $0.288 \pm 0.077$ & $-0.058 \pm 0.017$ & ext \\
\hline NFW & $3 \mathrm{D}$ & rel & $4.051 \pm 0.067$ & $0.197 \pm 0.093$ & $-0.084 \pm 0.020$ & ext \\
\hline NFW & $3 \mathrm{D}$ & srel & $4.704 \pm 0.151$ & $0.519 \pm 0.187$ & $-0.054 \pm 0.039$ & ext \\
\hline NFW & $2 \mathrm{D}$ & all & $3.580 \pm 0.040$ & $0.003 \pm 0.053$ & $0.051 \pm 0.013$ & ext \\
\hline NFW & $2 \mathrm{D}$ & rel & $3.813 \pm 0.050$ & $0.108 \pm 0.064$ & $-0.032 \pm 0.015$ & ext \\
\hline NFW & $2 \mathrm{D}$ & srel & $4.380 \pm 0.113$ & $0.420 \pm 0.137$ & $-0.052 \pm 0.030$ & ext \\
\hline gNFW & $3 \mathrm{D}$ & all & $3.671 \pm 0.055$ & $0.050 \pm 0.086$ & $0.101 \pm 0.019$ & ext \\
\hline gNFW & $3 \mathrm{D}$ & rel & $4.091 \pm 0.068$ & $0.057 \pm 0.098$ & $0.018 \pm 0.021$ & ext \\
\hline gNFW & $3 \mathrm{D}$ & srel & $4.646 \pm 0.152$ & $0.457 \pm 0.195$ & $-0.023 \pm 0.040$ & ext \\
\hline gNFW & $2 \mathrm{D}$ & all & $4.088 \pm 0.047$ & $-0.228 \pm 0.055$ & $0.164 \pm 0.014$ & ext \\
\hline gNFW & $2 \mathrm{D}$ & rel & $4.261 \pm 0.055$ & $-0.159 \pm 0.063$ & $0.071 \pm 0.015$ & ext \\
\hline gNFW & $2 \mathrm{D}$ & srel & $4.660 \pm 0.117$ & $0.138 \pm 0.129$ & $0.022 \pm 0.029$ & ext \\
\hline Einasto & $3 \mathrm{D}$ & all & $3.407 \pm 0.055$ & $0.040 \pm 0.092$ & $0.088 \pm 0.020$ & ext \\
\hline Einasto & $3 \mathrm{D}$ & rel & $3.805 \pm 0.068$ & $0.088 \pm 0.104$ & $-0.007 \pm 0.022$ & ext \\
\hline Einasto & $3 \mathrm{D}$ & srel & $4.366 \pm 0.151$ & $0.470 \pm 0.204$ & $-0.046 \pm 0.043$ & ext \\
\hline Einasto & $2 \mathrm{D}$ & all & $3.617 \pm 0.034$ & $0.070 \pm 0.049$ & $0.103 \pm 0.012$ & ext \\
\hline Einasto & $2 \mathrm{D}$ & rel & $3.729 \pm 0.041$ & $0.020 \pm 0.060$ & $0.028 \pm 0.014$ & ext \\
\hline Einasto & $2 \mathrm{D}$ & srel & $4.151 \pm 0.096$ & $0.352 \pm 0.126$ & $0.012 \pm 0.028$ & ext \\
\hline NFW & $2 \mathrm{D}$ & all & $3.978 \pm 0.055$ & $0.651 \pm 0.073$ & $-0.214 \pm 0.018$ & sl \\
\hline NFW & $2 \mathrm{D}$ & rel & $4.200 \pm 0.068$ & $0.593 \pm 0.090$ & $-0.185 \pm 0.021$ & sl \\
\hline NFW & $2 \mathrm{D}$ & srel & $4.658 \pm 0.150$ & $0.781 \pm 0.189$ & $-0.124 \pm 0.041$ & sl \\
\hline gNFW & $2 \mathrm{D}$ & all & $4.338 \pm 0.056$ & $0.276 \pm 0.073$ & $-0.060 \pm 0.018$ & sl \\
\hline gNFW & $2 \mathrm{D}$ & rel & $4.571 \pm 0.069$ & $0.310 \pm 0.089$ & $-0.053 \pm 0.020$ & sl \\
\hline gNFW & $2 \mathrm{D}$ & srel & $4.892 \pm 0.152$ & $0.558 \pm 0.187$ & $-0.059 \pm 0.041$ & sl \\
\hline Einasto & $2 \mathrm{D}$ & all & $3.774 \pm 0.053$ & $0.465 \pm 0.080$ & $-0.128 \pm 0.019$ & sl \\
\hline Einasto & $2 \mathrm{D}$ & rel & $3.961 \pm 0.066$ & $0.489 \pm 0.098$ & $-0.128 \pm 0.022$ & sl \\
\hline Einasto & $2 \mathrm{D}$ & srel & $4.317 \pm 0.147$ & $0.684 \pm 0.208$ & $-0.102 \pm 0.045$ & sl \\
\hline NFW & $2 \mathrm{D}$ & all & $4.105 \pm 0.100$ & $0.668 \pm 0.341$ & $-0.160 \pm 0.108$ & xray \\
\hline gNFW & $2 \mathrm{D}$ & all & $4.228 \pm 0.138$ & $0.376 \pm 0.458$ & $-0.080 \pm 0.145$ & xray \\
\hline Einasto & $2 \mathrm{D}$ & all & $3.880 \pm 0.119$ & $-0.017 \pm 0.425$ & $-0.035 \pm 0.137$ & xray \\
\hline
\end{tabular}

Notes. The results are listed for the concentration-mass measurements based on the NFW, gNFW, and Einasto models. First column: fitting model; second column: $3 \mathrm{D}$ or $2 \mathrm{D}$ analysis; third column: relaxation state (all = full sample; rel = relaxed; srel = super-relaxed); columns 3, 4, 5: $c-M-z$ parameters (see Equation (26)); column 6: selection function (ext = extended sample, no selection applied except that based on the relaxation state; $\mathrm{sl}=$ strong lensing selection; $x$ ray $=\mathrm{X}$-ray selection). The parameters of the $2 \mathrm{D} c-M-z$ relation for the extended sample are taken from J. Vega et al. (in preparation).

lowest redshift investigated in this work $(z=0.250)$. Each circle corresponds to a halo, and the solid, dashed, and dotted lines indicate the best-fit $c-M-z$ relations for the full, relaxed, and super-relaxed samples. At fixed mass, the distribution of NFW halo concentrations is reasonably well fitted by a lognormal distribution and have a standard deviation $\sigma_{c} \sim 0.25$, compatible with the findings of several previous works (see e.g., Dolag et al. 2004). The concentrations derived from the gNFW and Einasto fits are characterized by a larger scatter. In all cases we find that the dependence of the concentration on mass is very shallow. For the NFW profile, $c \propto M^{-0.057 \pm 0.017}$ for the full sample. Instead, for the gNFW and the Einasto profiles, the logarithmic slope of the $c-M$ relation is slightly positive. For the relaxed and super-relaxed halos, all of the $c-M$ relations have logarithmic slopes that are negative or consistent with zero. As expected, we find that the more relaxed the halos are, the higher their concentrations (Zhao et al. 2009; Giocoli et al. 2012b). This result holds regardless of the fitting function. At the lowest masses, the relative change in typical concentrations between the full and the relaxed (or super-relaxed) samples is larger for the gNFW and Einasto fits. In fact, we find that a larger fraction of small-mass unrelaxed halos are fitted with lower concentrations using these two fitting models than with the NFW profile. These halos are responsible for the positive logarithmic slope of the $c-M$ relation when fitting with the gNFW or Einasto profiles.
As can be seen from the $B$ parameters listed in Table 2, the normalization of the $3 \mathrm{D} c-M$ relation has an almost negligible redshift dependence for the full sample. For example, in the case of the NFW profile, $c \propto(1+z)^{-0.29 \pm 0.08}$. For the gNFW and Einasto profiles, the redshift evolution is even shallower. We notice, however, that the dependence of the concentration on redshift appears to be stronger for the most relaxed systems. In particular, for the super-relaxed halos, we find $B \sim 0.52$, regardless of the fitting function.

\subsubsection{The NFW Concentration-Mass Relation}

There are several parameterizations of the $c-M$ relation in the literature, mostly derived from fitting simulated halos using NFW profiles. In the upper panel of Figure 10, we show the NFW $c-M-z$ relation derived from the $3 \mathrm{D}$ analysis for the whole sample of MUSIC-2 halos (solid lines). We use different colors to show how the relation evolves with redshift. We find a rather shallow dependence of the concentrations on mass and redshift. Over the mass range $\left[4-12 \times 10^{14} h^{-1} M_{\odot}\right]$, the concentrations vary by less than $10 \%$, decreasing as a function of mass as $M^{-0.058 \pm 0.017}$. The amplitude of the $c-M$ relation scales with redshifts as $(1+z)^{-0.29 \pm 0.08}$. Other authors find that the $c-M$ relation of massive halos is rather flat. For example, Zhao et al. (2009), studying an ensemble of numerical simulations in the context of various cosmological models, find that the 

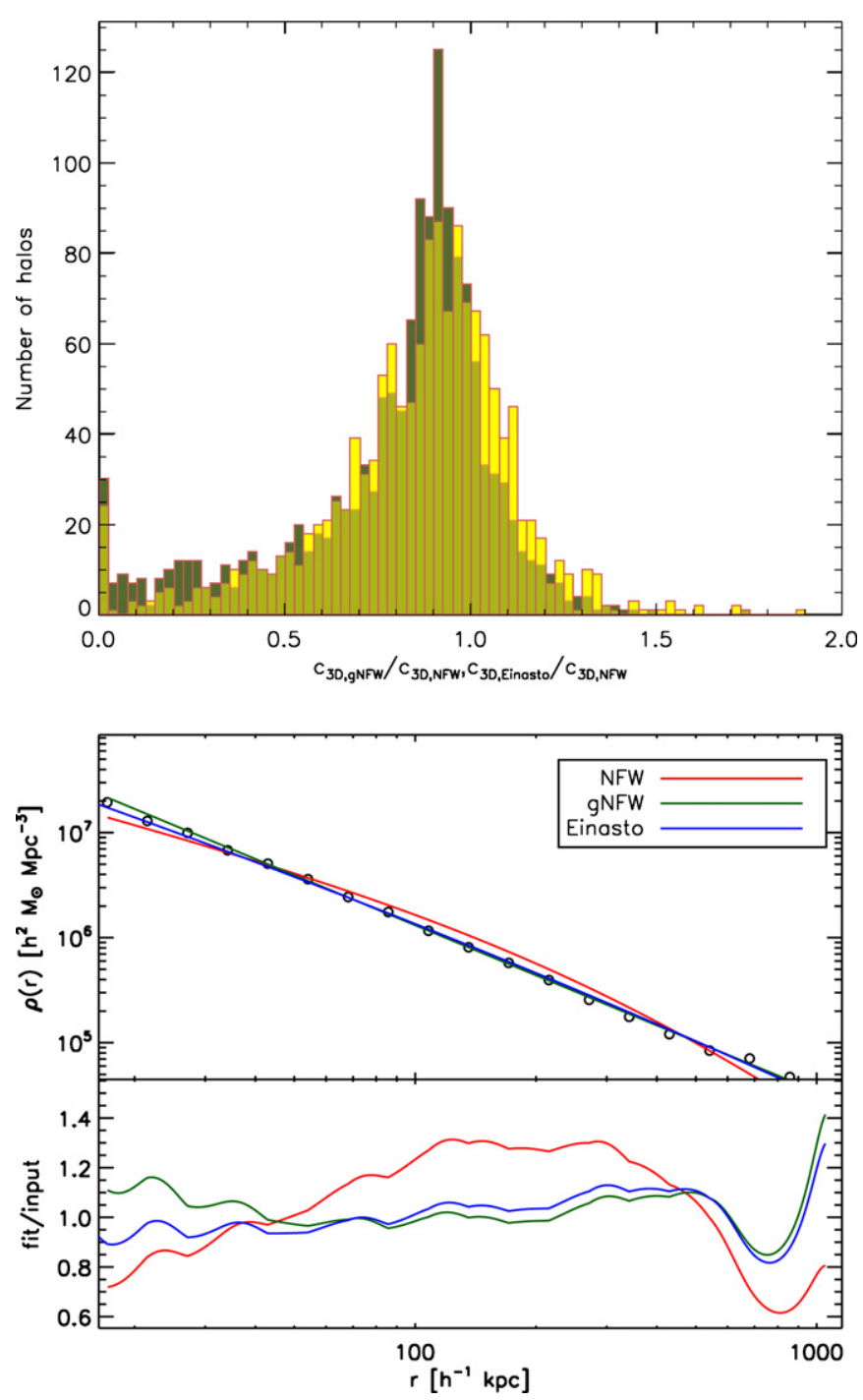

Figure 8. Upper panel: distributions of concentration ratios $c_{3 \mathrm{D}, \mathrm{gNFW}} / c_{3 \mathrm{D}, \mathrm{NFW}}$ (yellow histogram) and $c_{3 \mathrm{D} \text {,Einasto }} / c_{3 \mathrm{D}, \mathrm{NFW}}$ (green histogram). Bottom panel: example of a density profile for which the Einasto and gNFW concentrations are nearly zero. The halo profile is indicated by the open circles, and the best-fit NFW, gNFW, and Einasto profiles are given by the red, green, and blue lines, respectively. In the lower subpanel we show the ratio between the best fit and the input profiles.

(A color version of this figure is available in the online journal.)

concentration is strongly correlated with the age of the universe when the halo progenitor on the mass accretion history first reaches $4 \%$ of its current mass. According to this correlation, they find that the concentration is nearly constant for halos with mass $M \gtrsim 10^{14} h^{-1} M_{\odot}$. They also predict a very shallow redshift evolution of the $c-M$ relation. In a recent work, De Boni et al. (2013) also find concentrations that scale with mass and redshift, similar to our results. Their concentrations scale with mass and redshift as $M^{-0.07}$ and $(1+z)^{-0.26}$, respectively.

The normalization of our $c-M-z$ relation is higher than that found by some other authors, like De Boni et al. (2013; dotdashed lines in the upper panel of Figure 10) or Duffy et al. (2008). In these cases, the differences can be explained in terms of different cosmological settings. For example, De Boni et al. (2013) analyze halos evolved in the framework of a WMAP3 cosmological model and adopt a rather small normalization of the matter power spectrum, $\sigma_{8}=0.72$. If we consider other

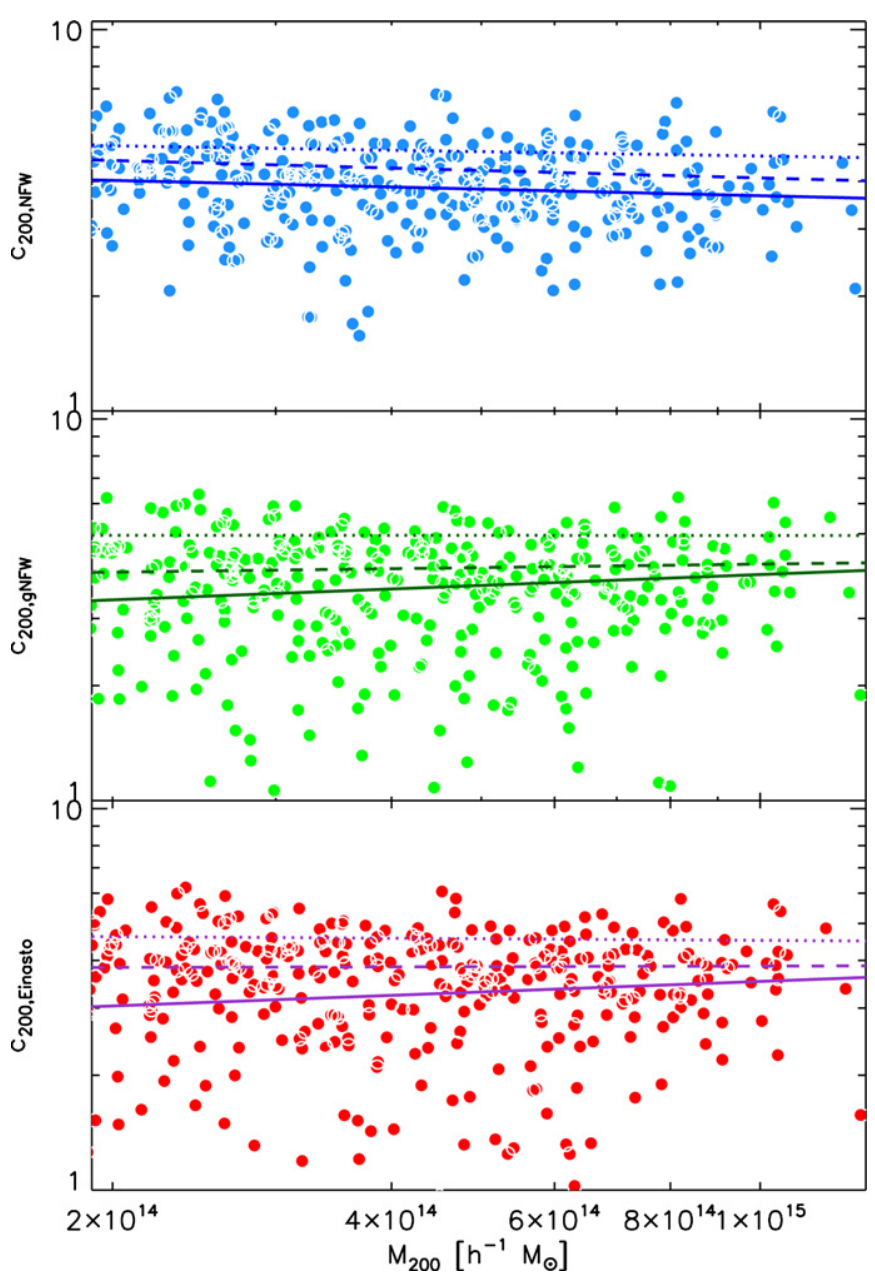

Figure 9. Concentration-mass measurements at $z=0.250$. The results are shown for the full sample (filled circles). The upper, middle, and bottom panels refer to the NFW, gNFW, and Einasto fits, respectively. In each panel, we show the best-fit $c-M-z$ relations for the full, relaxed, and super-relaxed samples (solid, dashed, and dotted lines, respectively).

(A color version of this figure is available in the online journal.)

analyses in the literature in the context of WMAP7 normalized cosmologies, the agreement is much better. For example, the $c-M$ relation that best fits our data at low redshift is in rather good agreement with the results of Bhattacharya et al. (2013) for nonrelaxed halos. For comparison, their $c-M$ relation is overplotted in the upper panel of Figure 10 (dashed lines). At $z=0.250$, the concentrations we measure at a given mass are only $\lesssim 6 \%$ higher than found by Bhattacharya et al. (2013). However, their $c-M$ relation has a stronger redshift evolution. Between $z=0.250$ and $z=0.667$, their concentrations at a fixed mass decrease by $\sim 17 \%$, while ours vary only by $\sim 10 \%$.

Potentially important differences between this work and Bhattacharya et al. (2013) are (1) our simulations include baryons, while the halos studied by Bhattacharya et al. (2013) are made only of dark matter; (2) our analysis focuses on a limited mass range, and the volume we sample is smaller than in the simulations employed by Bhattacharya et al. (2013); (3) the mass resolution of our simulations is roughly two orders of magnitude better; (4) Bhattacharya et al. (2013) fit their halos over a different radial range, $\left[0.1-1 r_{\text {vir }}\right]$ versus [0.02-1 $\left.r_{200}\right]$; and, finally, (5) Bhattacharya et al. (2013) fit the mass profiles instead of the density profiles, as we do. Given that our simulations are nonradiative, it is unlikely that the 


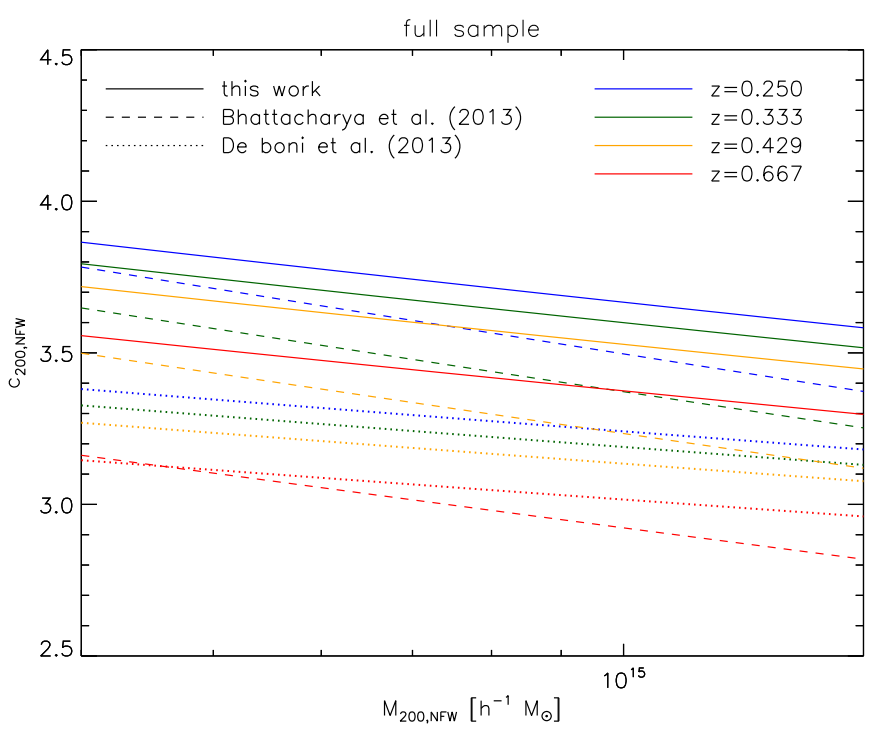

relaxed \& super-relaxed

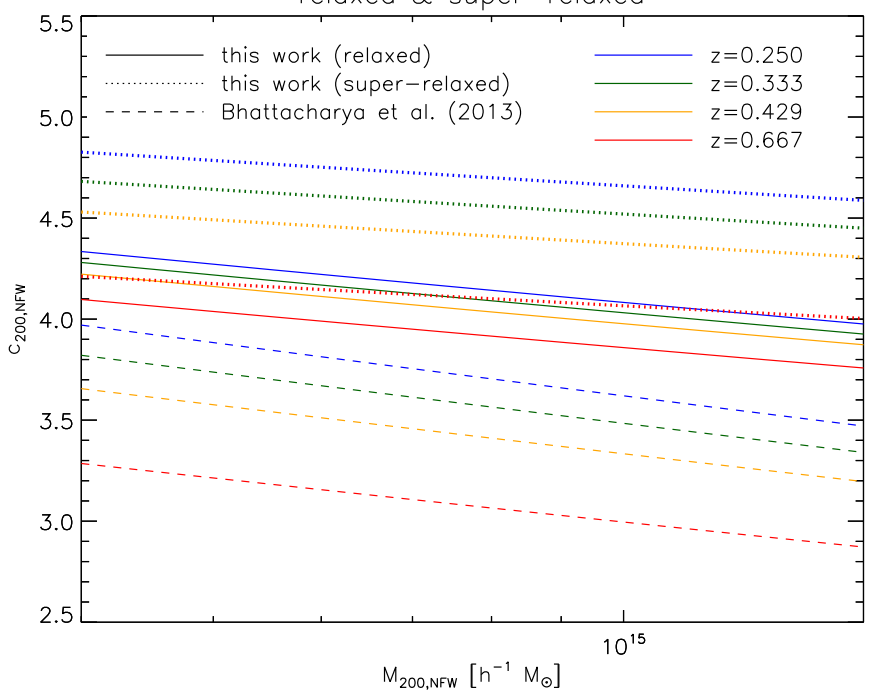

Figure 10. Concentration-mass relation and its redshift evolution as obtained from fitting the halo density profiles with the NFW model. The results of this analysis are compared with the recent work of Bhattacharya et al. (2013; dashed lines) and De Boni et al. (dotted lines in the upper panel). The redshift evolution is illustrated by different colors. The upper and bottom panels show the results for the whole sample and for the subsamples of relaxed and super-relaxed halos. Note that Bhattacharya et al. (2013) only distinguish between relaxed and unrelaxed halos.

(A color version of this figure is available in the online journal.)

differences between the $c-M$ relations arise from baryonic effects. De Boni et al. (2013) show that concentrations are higher by $5 \%-15 \%$ in radiative simulations compared to dark matter-only simulations. This result, however, was obtained using hydrodynamical simulations that are known to suffer from the overcooling problem. It has been shown by other authors that halos in adiabatic simulations develop density profiles pretty similar to those of pure dark matter halos (Killedar et al. 2012). The different mass range, volume, and resolution of the simulations may have a larger impact on the results. Because our halos are sampled with a larger number of particles, the profiles are better resolved. Thus, the measurements of the individual concentrations should be more robust and allow us to resolve smaller radial scales. On the other hand, because their volume is bigger, Bhattacharya et al. (2013) have a larger number of massive halos to constrain the $c-M$ relation at the cluster scales. In contrast, Bhattacharya et al. (2013) fit halos over three orders of magnitude in mass. It may be possible that the strong redshift evolution of their $c-M$ relation is driven by the smallest halos. Overall, it is likely that the higher normalization of our $c-M$ is largely due to the better resolution of the MUSIC-2 sample compared to the simulation sets used in Bhattacharya et al. (2013).

The bottom panel in Figure 10 shows another comparison between our best-fit NFW $c-M-z$ relation and the results of Bhattacharya et al. (2013). The solid and the dotted lines show our relations for relaxed and the super-relaxed samples, respectively. The most striking difference from Bhattacharya et al. (2013) (dashed lines) is that we find a much stronger dependence of concentration on the halo dynamical state. While the normalization of our $c-M-z$ relation increases by $\sim 10 \%$ between the full and relaxed samples, Bhattacharya et al. (2013) find that concentrations of relaxed halos increase only by $\sim 3 \%$.

\subsubsection{The c-M Relation and Temperature Selection}

The CLASH relaxed sample is composed of clusters with $\mathrm{X}$-ray temperatures larger than $5 \mathrm{keV}$. Even if the observational sample is not complete, it is interesting to check whether a selection based on temperature may lead to a biased concentration-mass relation. Once more, we stress that the description of the gas physical processes is not sufficiently accurate in these nonradiative simulations to reproduce several observed X-ray properties of clusters. Thus, we do not aim to draw quantitative conclusions here. Rather, we are interested in understanding in which directions a temperature selection would change the results.

Under the assumption of self-similarity, expected in the case that cluster properties and correlations between them are determined by gravity alone and that clusters are in virial equilibrium, the mass should scale as $\propto T^{2 / 3}$, thus implying that both selections based on mass and temperature should lead to the same samples. Unfortunately, as we discussed earlier, a large fraction of halos is still far from being in equilibrium. As a result, the scatter around the $M-T$ relation is large (see, e.g., Rasia et al. 2011). At fixed mass, the halos with the lowest temperatures are also less concentrated, implying that in introducing a temperature cut we would exclude the least concentrated halos, thus increasing the average concentration of the sample.

In Figure 12, we illustrate the effects of the selection by showing the distributions of the halos at $z=0.250$ in the $c-M$ plane. The upper panel refers to all halos in the MUSIC-2 sample. The red circles indicate those halos that have a massweighted temperature $T_{\mathrm{mw}}>5 \mathrm{keV}$. As expected, the hottest halos are the most massive in the sample. At the largest masses, almost all halos pass the temperature cut. However, if we consider less-massive objects, we notice that there is an increasing fraction of halos that are not hot enough to be selected. The halos that do not pass the cut have likely experienced a recent merger and are therefore characterized by small concentrations. The accretion of a smaller (and colder) object decreases temporally the measured temperature before the shock heats the intracluster medium. This implies that the small-mass halos in the temperature-selected sample have concentrations above the average of the full sample. Given that it affects the sample composition in a way that depends on the mass, the temperature selection thus changes the overall slope of the $c-M$ relation. The best linear fits to the data in 

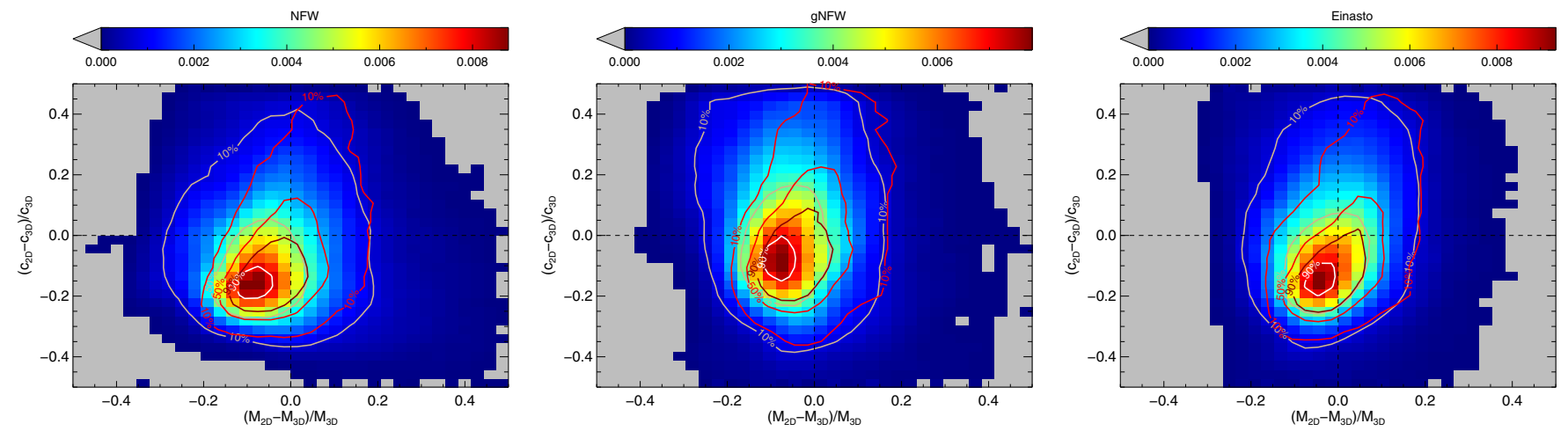

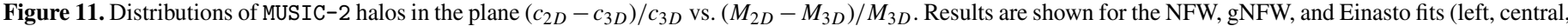

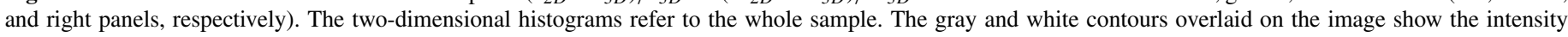
levels corresponding to $10 \%, 50 \%$, and $90 \%$ of the probability peak. The red contours correspond to the same levels for the distributions of the relaxed halos.

(A color version of this figure is available in the online journal.)

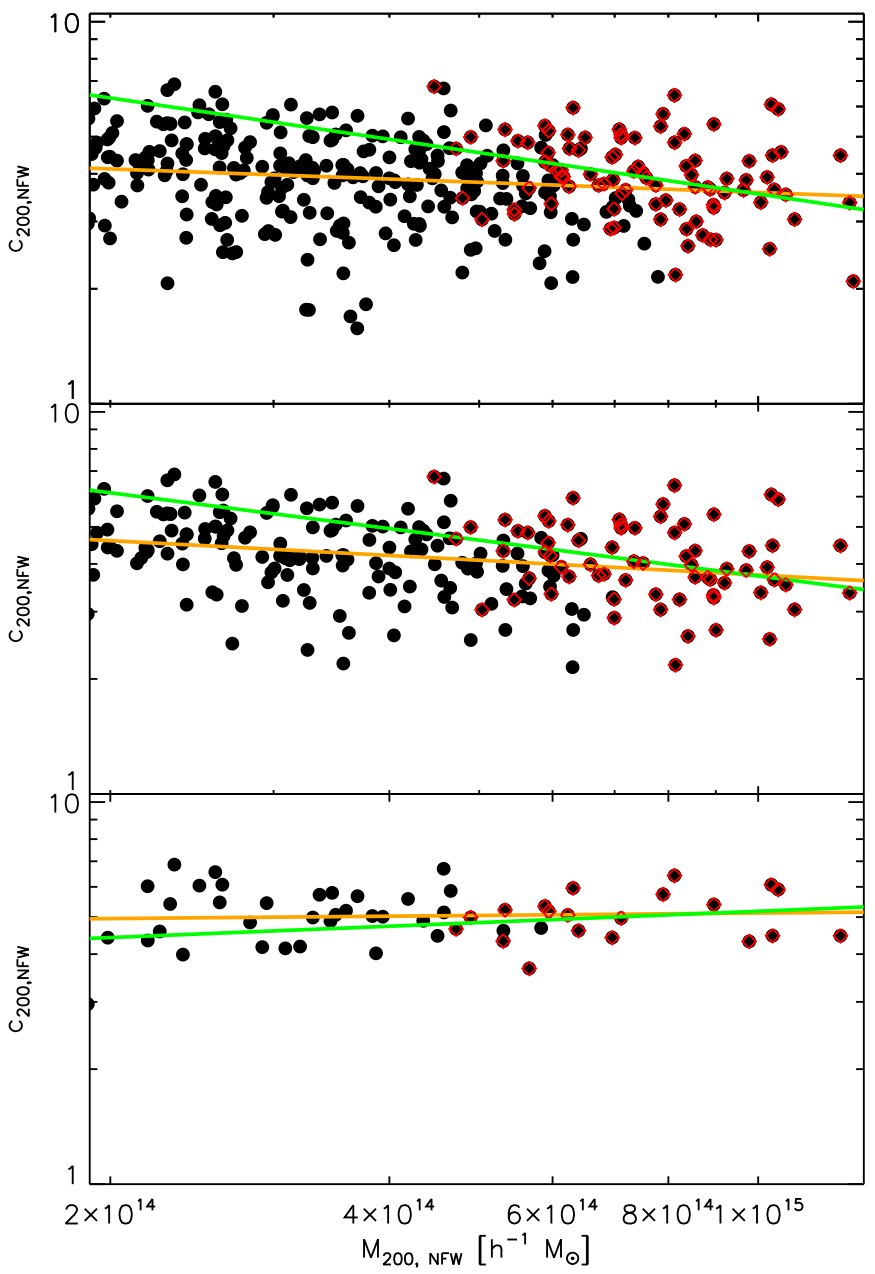

Figure 12. Effects of temperature selection on the $c-M$ relation. The black data points show the NFW 3D concentrations vs. masses for all halos in the MUSIC-2 sample at $z=0.250$. The red circled data points indicate the halos with mass-weighted temperature $T>5 \mathrm{keV}$. While all (relaxed and unrelaxed) halos are used in the upper panel, the middle and the bottom panels show the results for the relaxed and super-relaxed halos only. The orange and green solid lines are the best-fit $c-M$ relations to the data points before and after applying the temperature cut.

(A color version of this figure is available in the online journal.) the $\log M-\log c$ space are given by the orange and green lines, which refer to the samples without and with temperature cuts applied.

However, the differences between the $c-M$ relations of massand temperature-selected samples become negligible when additional filtering is applied based on the relaxation state. Given that the temperature selection mainly affects the fraction of unrelaxed halos in the sample, there are not significant differences between the mass and temperature selections when the unrelaxed halos are discarded a priori. Thus, the $c-M$ relations of relaxed and super-relaxed halos are less sensitive to the temperature selection. This is shown in the middle and in the bottom panels of Figure 12, which refer to the relaxed and super-relaxed halos, respectively.

\subsubsection{The Concentration-Mass Relation in $2 D$}

The 2D concentration-mass relation of the MUSIC-2 halos will be discussed in detail in an upcoming paper ( $\mathrm{J}$. Vega et al., in preparation). We briefly summarize some properties of this $c-M$ relation that are relevant for the following discussion. Projection effects do affect concentrations, which are generally found to be smaller than in 3D. This effect of triaxiality, also discussed in Giocoli et al. (2012a), is illustrated in Figure 11, where we show the distribution of the MUSIC-2 halos in the $\left(c_{2 D}-c_{3 D}\right) / c_{3 D}$ versus $\left(M_{2 D}-M_{3 D}\right) / M_{3 D}$ plane. The $2 \mathrm{D}$ histograms show that regardless of the fitting model, the masses and concentrations derived from fitting the surface-density profiles tend to be smaller than measured from fitting the density profiles. The trend is in qualitative agreement with the findings of Giocoli et al. (2012a), although the amplitude of both the concentration and mass biases found here is smaller. The white contours overlaid on the 2D histograms show the intensity levels corresponding to $10 \%, 50 \%$, and $90 \%$ of the peaks of the distributions. The red contours indicate the same intensity levels for the subsample of super-relaxed halos. As explained in Section 5.3, the bias is reduced for the relaxed halos because these systems are typically more spherical than the unrelaxed halos.

The best-fit parameters of the 2D $c-M-z$ relation are listed in Table 2. The relations for halos at $z=0.250$ are given by the solid lines in Figure 13. Interestingly, the $c-M$ relation is very flat and characterized by an inverted slope compared to the $c-M$ relation in $3 \mathrm{D}$. This suggests that the $2 \mathrm{D}$ concentrations underestimate the 3D ones more significantly at the lowest than at the highest masses. One possible explanation is that the halo triaxiality is somehow biased below the completeness limits 


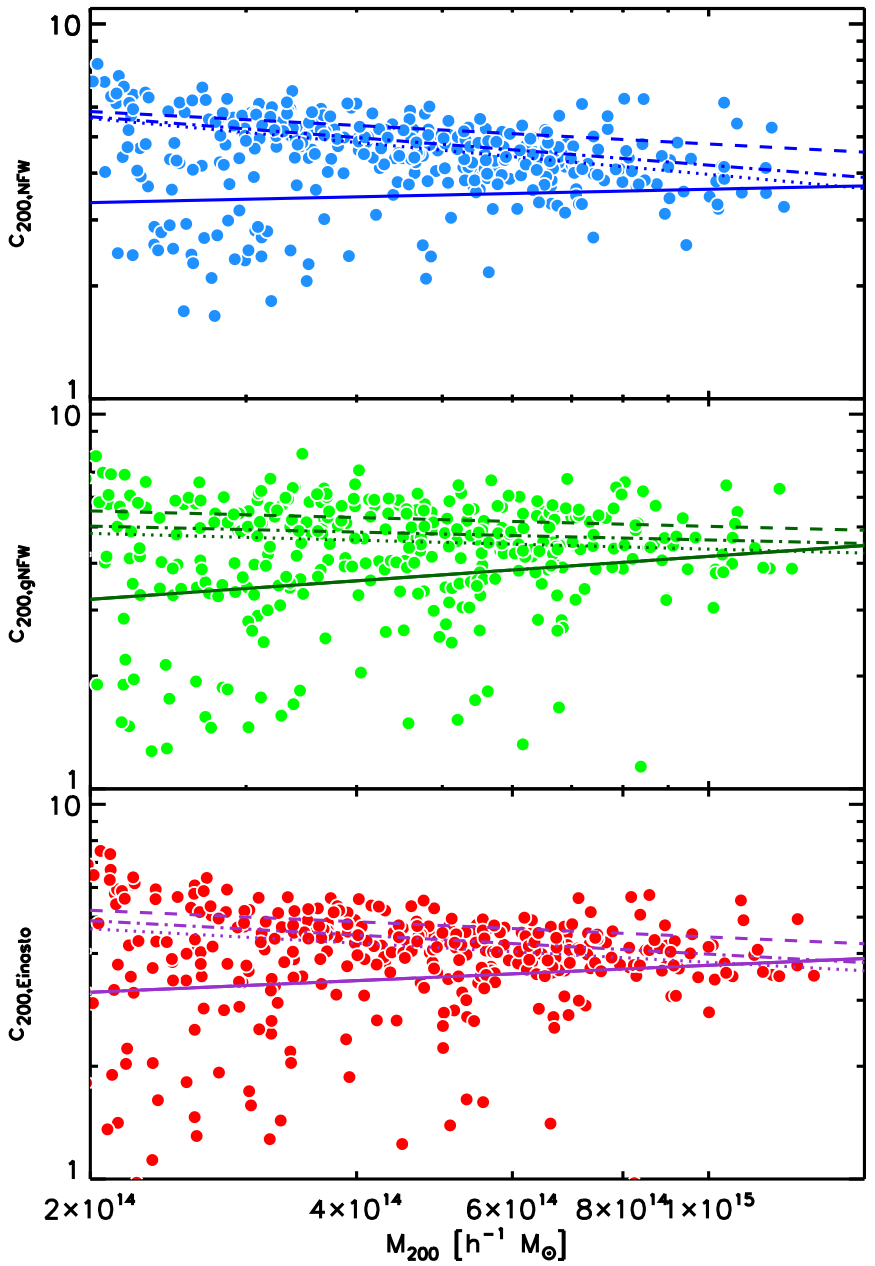

Figure 13. Concentration-mass relation for strong-lensing halos at $z=0.250$. The lines indicate the results obtained for the halos in the full, relaxed, and super-relaxed samples (dotted, dot-dashed, and dashed lines, respectively). For comparison, the 2D $c-M$ relation derived for the full sample including nonstrong lenses is shown by the solid line. The colored circles correspond to the projections capable of producing critical lines for $z_{s}=2$. The upper, middle, and bottom panels refer to the NFW, gNFW, and Einasto fits.

(A color version of this figure is available in the online journal.)

listed in Table 1 . However, we checked that the $c-M$ relation obtained only from halos above the completeness limits does not differ significantly from what we obtain using the extended sample. However, the constraints on its slope are obviously weakened. In addition, we notice that Giocoli et al. (2012a) also find indications for a $2 \mathrm{D}$ concentration bias that decreases as a function of mass. This will be discussed in J. Vega et al. (in preparation).

\section{THE CONCENTRATION-MASS RELATION OF CLASH-LIKE CLUSTERS}

We can now discuss how different cluster selection methods impact the $c-M-z$ relation. We will start with the $c-M-z$ relation of strong-lensing (SL) galaxy clusters. Then we investigate the $c-M-z$ relation obtained by selecting halos on the basis of their X-ray morphology. The results of this analysis are compared to the observations in Merten et al. (2014) and Umetsu et al. (2014).

\subsection{The $c-M-z$ Relation of Strong-lensing Halos}

As explained above, the CLASH cluster sample is composed of 25 galaxy clusters, of which only five were selected on the basis of their SL strength. The remaining 20 clusters are not SL selected, and they were chosen on the basis of their $\mathrm{X}$-ray morphology. We will discuss this selection method in the next section. Nevertheless, SL features (multiple images and arcs) have been securely detected in all CLASH clusters except RXJ 1532.8+3021. The analyses of these SL features have allowed the creation of detailed lens models and the measurement of their Einstein radii (Zitrin et al. 2014). The Einstein radii for sources at redshift $z_{\mathrm{s}}=2$ are within the range 5-55 arcsec.

We construct the $c-M-z$ relation of SL galaxy clusters by selecting those projections where we measure an Einstein radius compatible with those measured in the CLASH sample $\left(\theta_{E}>5^{\prime \prime}\right)$. As explained, the Einstein radius is defined as in Equation (18).

In Figure 13, we show the concentration-mass relations at $z=0.250$ derived from SL halos in the MUSIC-2 sample. The relations are displayed for the NFW, gNFW, and Einasto models (upper, middle, and bottom panels). The corresponding parameters are listed in Table 2. The dotted, dashed, and dot-dashed lines indicate the relations obtained for the full, the relaxed, and the super-relaxed samples, respectively. For comparison, we show also the $c-M$ relation for the full sample, including also the nonstrong lenses, as discussed in Section 5.4.4. By requiring that the halos are strong lenses in their projections, we remove a large fraction of halos with low concentrations, obtaining relations characterized by a larger normalization. In particular, an increasingly larger number of halos of small mass are unable to produce an appreciable SL signal. By removing them from the initial catalog, we restore the negative logarithmic slope of the $c-M$ relation. Because of this selection, the concentration scales with mass as $c \propto M^{-0.214 \pm 0.018}$. This result is in very good agreement with the theoretical predictions of Giocoli et al. (2013) and Oguri et al. (2012), who estimated the lensing bias of the $c-M$ via semianalytic calculations employing triaxial halos. For the gNFW and Einasto models, the concentration-mass relations are slightly flatter.

Even for the SL halos, the normalization of the $c-M-z$ relation depends on the relaxation state. The most relaxed systems have the largest concentrations. The differences between the $c-M-z$ relations of relaxed and unrelaxed halos are smaller than found earlier for the whole sample including nonstrong lenses, though. This is because in the SL-selected sample the fraction of relaxed and super-relaxed halos is pretty high. At $z=0.250$, about $75 \%$ of the SL projections belong to relaxed halos. The fraction of super-relaxed halos in this sample is $\sim 27 \%$. At $z=0.667$ the fractions of relaxed and super-relaxed halos are $\sim 60 \%$ and $\sim 13 \%$, respectively.

Finally, we find that the redshift evolution of the $c-M$ relation of SL halos is stronger than for non-SL halos. The values for the $B$ parameters listed in Table 2 are in the range [0.48-0.64] for the three fitting models.

\subsection{The $c-M-z$ Relation of $X$-ray-selected Halos}

We discuss now the impact of the X-ray selection on the concentration-mass relation. In particular, we discuss the expectations for halos selected so as to resemble the X-ray morphologies of the clusters in the CLASH X-ray-selected sample. The results shown here are based on the analysis of three 


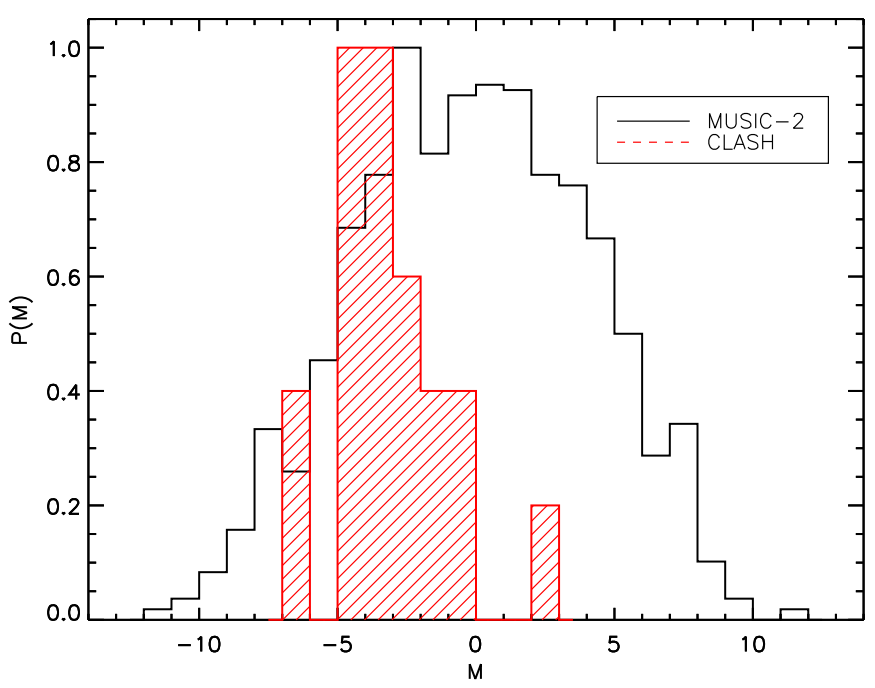

Figure 14. Distributions of regularity parameters $M$ in the MUSIC-2 (black histogram) and in the CLASH sample (red histogram).

(A color version of this figure is available in the online journal.)

projections per halo, and the halos considered are those with 3D mass above the completeness limits given in Table 1. The restriction of the analysis to this smaller sample of simulated halos was dictated by the large computational time required to produce the X-ray-simulated observations. The mass range covered by these simulations is however representative of the mass range of the CLASH clusters (see both Merten et al. 2014 and Umetsu et al. 2014).

As explained in Section 4.1, the X-ray morphology is measured by means of five morphological parameters. They can be combined to quantify the degree of regularity of the halos, as shown in Equation (24). The regularity parameters of the CLASH clusters, as measured in their X-ray images, are listed in Table 3. In Figure 14, we show their distribution (red histogram), and we compare it to the distribution of the regularity parameters in the MUSIC-2 sample (black histogram). The histograms have been normalized to have the same peak value. As it emerges from these distributions, the CLASH clusters have quite typical regularity parameters in the simulations. With the exception of MACSJ 1206.2-0847, the clusters in the CLASH $\mathrm{X}$-ray-selected sample have negative $M$ parameters, indicating that they are more regular than the mean of the simulations. Even in the case of MACSJ 1206.2-0847, other works based on different analyses find that this system is not likely to be perturbed by significant substructures (Lemze et al. 2013; Biviano et al. 2013). This is expected given that these clusters were selected on the basis of their X-ray regularity. On the other hand, the comparison shows that their regularity is not extreme, in the sense that there are several simulated halos with regularity parameters exceeding the values for the CLASH clusters. In fact, the simulated sample has a tail of low $M$ values extending well beyond those of the CLASH clusters.

In the upper panel of Figure 15, we show that the concentration inferred from the analysis of the 2D mass distributions is correlated with the regularity parameter $M$. The red, green, and blue circles refer to unrelaxed, relaxed, and super-relaxed halos. The correlation was evaluated by measuring the linear Pearson correlation coefficient $P$ between the $\log _{10} c_{200,2 D}$ and the $M$ values. It is stronger for the super-relaxed halos, for which we measure $P=-0.67$. For the relaxed and the full samples,
Table 3

Comparison between CLASH Clusters and X-Ray-Selected Halos

\begin{tabular}{|c|c|c|c|c|c|}
\hline Cluster & $z_{\text {sim }}$ & $z$ & $M$ & $\begin{array}{c}M_{200, X} \\
\left(10^{14} h^{-1} M_{\odot}\right)\end{array}$ & $c_{200, X}$ \\
\hline A383 & 0.250 & 0.188 & -6.49 & $8.52 \pm 1.47$ & $3.46 \pm 1.09$ \\
\hline A209 & 0.250 & 0.206 & -0.87 & $9.43 \pm 1.76$ & $4.09 \pm 0.94$ \\
\hline A1423 & 0.250 & 0.213 & -3.11 & $7.00 \pm 1.80$ & $4.60 \pm 1.12$ \\
\hline A2261 & 0.250 & 0.225 & -3.93 & $9.98 \pm 2.03$ & $3.76 \pm 1.00$ \\
\hline RXJ2129+0005 & 0.250 & 0.234 & -3.70 & $6.12 \pm 2.71$ & $3.69 \pm 1.01$ \\
\hline A611 & 0.250 & 0.288 & -4.27 & $8.50 \pm 1.59$ & $3.12 \pm 1.43$ \\
\hline MS2137-2353 & 0.333 & 0.313 & -5.00 & $10.41 \pm 2.65$ & $4.38 \pm 1.11$ \\
\hline RXJ1532.8+3021 & 0.333 & 0.345 & -6.27 & $6.19 \pm 2.65$ & $3.73 \pm 1.11$ \\
\hline RXCJ2248-4431 & 0.333 & 0.348 & -1.56 & $11.50 \pm 3.33$ & $3.62 \pm 1.09$ \\
\hline MACSJ1115+0129 & 0.333 & 0.352 & -2.87 & $9.00 \pm 1.80$ & $3.07 \pm 1.45$ \\
\hline MACSJ1931-26 & 0.333 & 0.352 & -4.37 & $6.92 \pm 2.31$ & $3.91 \pm 1.05$ \\
\hline MACSJ1720+3536 & 0.429 & 0.391 & -4.12 & $7.50 \pm 1.92$ & $5.68 \pm 1.81$ \\
\hline MACSJ0429-02 & 0.429 & 0.399 & -3.50 & $8.05 \pm 1.81$ & $3.74 \pm 1.10$ \\
\hline MACSJ1206-08 & 0.429 & 0.439 & 2.29 & $8.62 \pm 1.96$ & $3.14 \pm 1.43$ \\
\hline MACSJ0329-02 & 0.429 & 0.450 & -2.90 & $7.31 \pm 1.89$ & $3.82 \pm 1.09$ \\
\hline RXJ1347-1145 & 0.429 & 0.451 & -2.79 & $11.47 \pm 4.20$ & $3.62 \pm 1.16$ \\
\hline MACSJ1311-03 & 0.429 & 0.494 & -3.44 & $6.09 \pm 2.31$ & $3.90 \pm 1.02$ \\
\hline MACSJ1423+24 & 0.667 & 0.545 & -4.10 & $5.71 \pm 2.49$ & $3.93 \pm 1.07$ \\
\hline MACSJ0744+39 & 0.667 & 0.686 & -1.56 & $7.00 \pm 1.93$ & $4.58 \pm 1.22$ \\
\hline
\end{tabular}

Notes. Column 1: cluster name; column 2: reference redshift in the simulations; column 3: true redshift of the CLASH cluster; column 4: regularity parameter $M$; column 5: mass range of X-ray-selected clusters in the simulation; column 6: mean NFW concentration of selected halos.

we obtain $P=-0.46$ and $P=-0.39$, respectively. The best linear fit between the two parameters is

$$
\log _{10} c_{200,2 D}=(0.598 \pm 0.009)-(0.019 \pm 0.002) \times M
$$

If we refer to the average of all halos in the simulations ( $M=0$ by construction), for negative values of $M$ we expect a positive concentration bias. Because the median value of the $M$ parameters of the CLASH clusters is $M_{\mathrm{CLASH}}=-3.44$, on the basis of Equation (27), we can give an estimate of the expected concentration bias for the CLASH X-ray-selected sample, which is

$$
\frac{c_{200, \mathrm{CLASH}}}{c_{200,2 D}(M=0)}=1.11 \pm 0.03 \text {. }
$$

An interesting question is whether this concentration excess compared to the full sample arises from the selection of purely relaxed halos. The answer is already contained in the upper panel of Figure 15: a selection based on the $M$ regularity parameter does not lead to the construction of a purely relaxed sample. Indeed, the left side of the diagram contains several red circles, indicating that unrelaxed halos can have $M<0$. The composition of samples selected by means of the $M$ parameter is shown in the bottom panel of Figure 15. The curves show the fractions of relaxed (R), nonrelaxed (NR), and super-relaxed (SR) halos in the samples with regularity parameter smaller than $M$. As indicated by the dotted and the solid black lines ( $\mathrm{R}$ and NR halos), the fraction of relaxed and unrelaxed halos is nearly constant as a function of $M$. Thus, there is no strong correlation between X-ray regularity and halo relaxation. In particular, we find that only $\sim 70 \%$ of the halos among those with $M<0$ are relaxed. ${ }^{37}$ The remainder $\sim 30 \%$ of the halos are unrelaxed. As stated, this composition is very similar to that of the full sample.

\footnotetext{
37 We remind that relaxed halos are identified by means of the criteria described in Section 5.2. By definition, super-relaxed halos are also included in this category.
} 

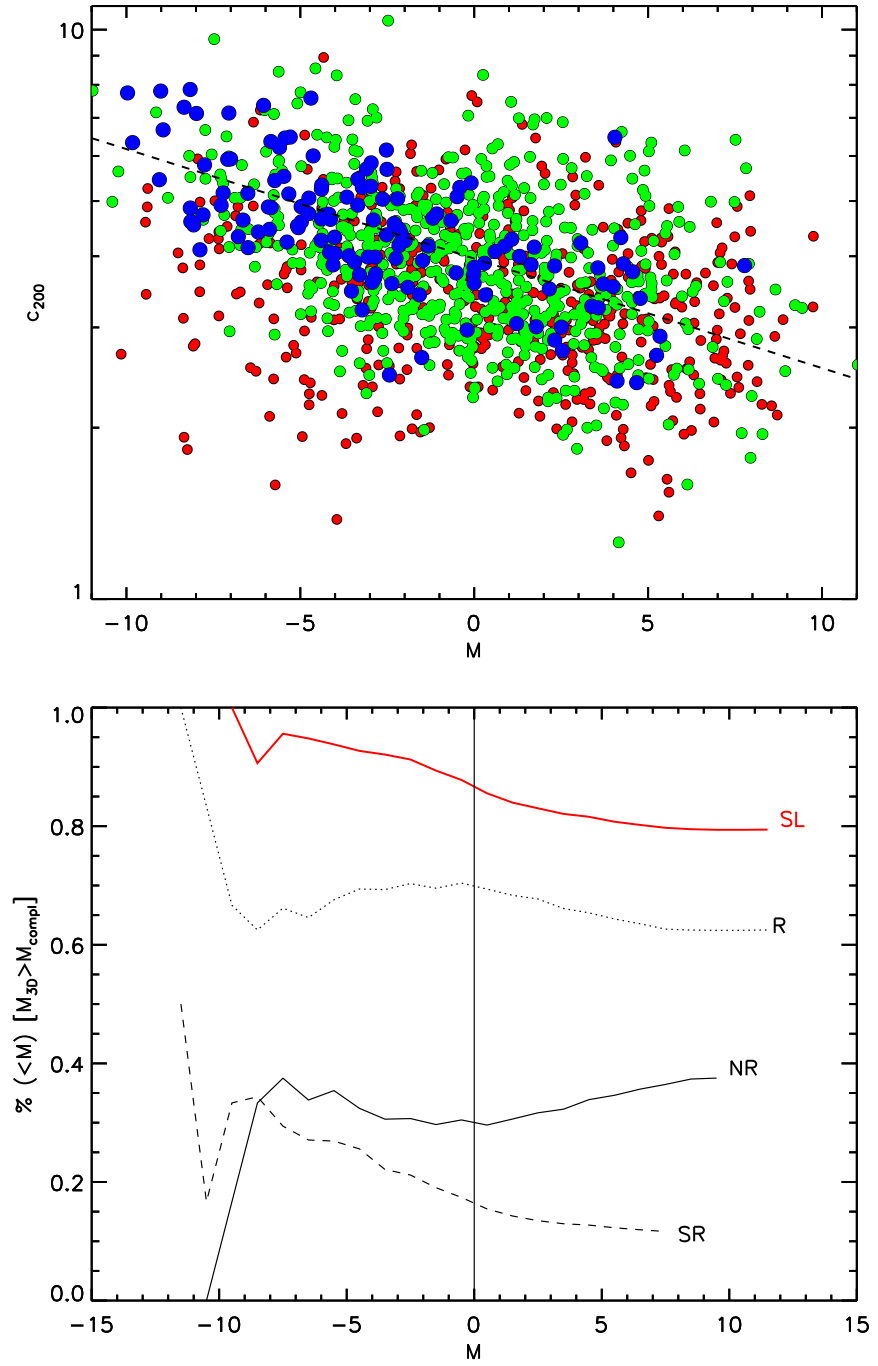

Figure 15. Upper panel: correlation between projected concentration and regularity parameter $M$. The red, green, and blue circles indicate the unrelaxed, relaxed, and super-relaxed halos, respectively. The dashed line shows the best linear fit between $\log _{10}\left(c_{200}\right)$ and $M$, obtained using all of the data points and given in Equation (27). Bottom panel: fraction of strong lensing (SL), unrelaxed (NR), relaxed (R), and super-relaxed (SR) halos in samples selected by means of the $M$ parameter.

(A color version of this figure is available in the online journal.)

In contrast, as indicated by the dashed line, the fraction of super-relaxed halos decreases as a function of $M$. Thus, in samples of clusters selected to have regular X-ray morphologies, we expect to have a larger fraction of super-relaxed halos. Because these typically have larger concentrations, we expect that the average concentration in an $M$-selected sample is higher than in the full sample.

The red solid line shows that the fraction of strong lensing (SL) halos in $M$-selected samples also decreases as a function of $M$. This trend reflects the correlation between concentration and regularity parameter. Because the halos are more concentrated, they more easily act as strong lenses. However, we notice that a correlation exists also between the concentrations and the $M$ parameters of the unrelaxed halos, although this is weaker than for the relaxed and super-relaxed halos. For unrelaxed halos, the linear Pearson coefficient is $P=-0.22$, indicating also that among these halos, those with a small $M$ parameter tend to have larger concentrations. In part, the classification of unrelaxed halos as regular is due to the different radial scales over which the

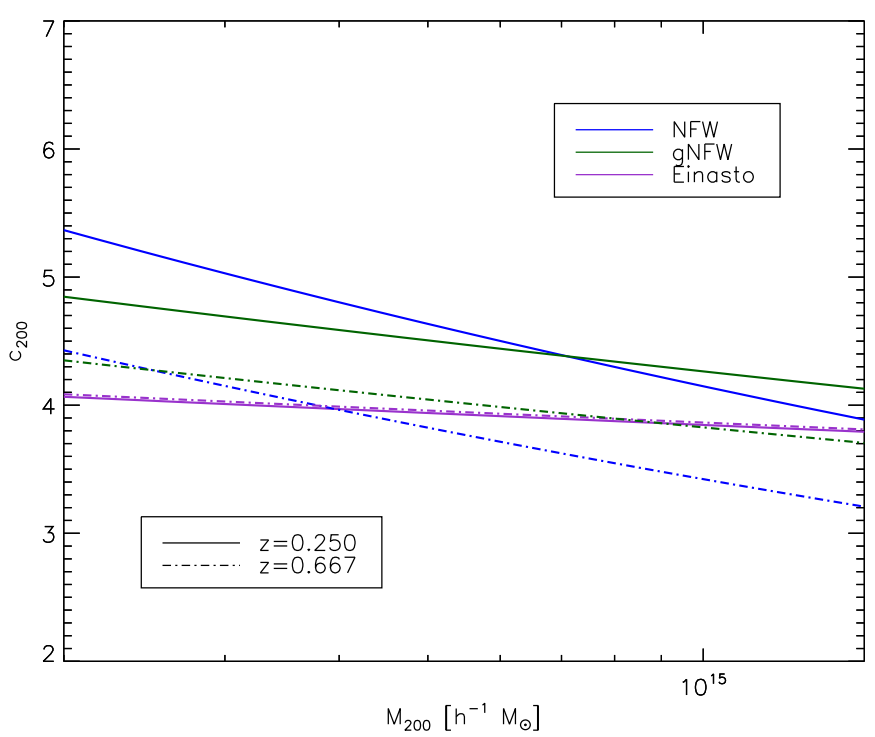

Figure 16. Concentration-mass relation at $z=0.250$ and $z=0.667$ for X-rayselected halos (solid and dot-dashed lines, respectively). The results are shown for the NFW, gNFW, and Einasto fitting models.

(A color version of this figure is available in the online journal.)

relaxation and the regularity are evaluated. Whereas the former is measured using all particles inside the virial radius, the second is meant to quantify the morphology of the cluster cores, within $500 \mathrm{kpc}$. A fraction of halos with regular X-ray morphologies have significant substructures outside $500 \mathrm{kpc}$, which implies that they are classified as unrelaxed. These substructures explain the low concentrations of those unrelaxed halos that have small $M$. However, for $\sim 42 \%$ of the unrelaxed halos with $M<0$, we do not find evidence for substructures outside the region where we carry over the X-ray morphological classification. These halos generally have $2 \mathrm{D}$ concentrations higher than the average of the sample, indicating that the selection based on X-ray morphology may lead to the inclusion of unrelaxed objects that are elongated along the line of sight. Such a sample would then be affected by a small orientation bias.

We use the $M$ parameters to create a sample of X-ray-selected halos. These halos are drawn from the full MUSIC-2 sample so as to reproduce the distribution of the $M$ parameters found for the CLASH clusters. In doing so, we take into account the masses and redshifts of the CLASH clusters. The masses are taken from Merten et al. (2014). A halo is selected if it has a suitable $M$ parameter and if the mass inferred from the $2 \mathrm{D}$ analysis is within $3 \sigma$ of the mass measured by CLASH. To account for the redshift distribution, given that the halos available for this analysis are simulated only at four redshifts, we create a match between each CLASH cluster and the nearest simulated redshift. The matches are listed in Table 3.

As explained earlier in the paper, the X-ray analysis is limited to three orthogonal lines of sight per halo. Given that many more projections are available in the 2D analysis of the MUSIC-2 halos, we can improve our statistical power by increasing the number of projections used. To do so, we identify the projections whose lines of sight are within $20 \mathrm{deg}$ of those selected in the $\mathrm{X}$-ray analysis.

Using the concentrations and masses inferred from the 2D analysis of the X-ray-selected projections, we fit the $c-M-z$ relation for our X-ray-selected sample. The relation is shown in Figure 16 for all of the fitting models employed in this study. The best-fit parameters are listed in Table 2. Overall, the 
$c-M-z$ relation for $\mathrm{X}$-ray-selected halos is in good agreement with the SL $c-M-z$ relation for a sample composed of both relaxed and unrelaxed halos. This is not surprising given that $\mathrm{X}$-ray-selected halos are frequently efficient strong lenses, with only $\sim 8 \%$ of them not having an extended critical line for sources at $z=2$. About $70 \%$ of the selected projections belong to relaxed halos. About $18 \%$ of them correspond to halos classified as super-relaxed. For the NFW model, we find that the concentrations scale with mass as $c \propto M^{-0.16 \pm 0.11}$, resulting in average concentrations that are intermediate between those predicted in 3D for relaxed and super-relaxed halos in the mass range $2 \times 10^{14} \lesssim M_{200} \lesssim 10^{15} h^{-1} M_{\odot}$.

Some differences between the fitting models are found with regards to the redshift evolution of the concentration-mass relation. For the NFW model, the $c-M-z$ relation is evolving strongly. The redshift dependence is shallower in the case of the gNFW model, and it is consistent with zero evolution for the Einasto profile.

\section{PREDICTIONS FOR INDIVIDUAL CLASH CLUSTERS}

Finally, we use the MUSIC-2 halos and their X-ray morphology to predict the concentrations of each individual CLASH cluster. As explained in Section 4.1, this is done using the parameter $C_{X}$, which measures the distance of each simulated halo from a given CLASH cluster in the multidimensional space defined by the X-ray morphological parameters. We select projections with $C_{X}<0.4$ to create the match.

Again, for each of the matched X-ray images, we include in our analysis the projections from nearby lines of sight. To be associated with a specific CLASH cluster, the halos must also have compatible masses and redshifts. For all of the CLASH clusters except CLJ 1226+3332, we could create associations with $\sim 10-200$ projections. CLJ 1226+3332 turned out not to have any counterpart in the simulated set. For this cluster, Merten et al. (2014) measured a large mass, $M_{200} \sim 1.5 \times 10^{15}$. The cluster is also at high redshift $(z=0.89)$, and because of the limited volume of the MultiDark cosmological box, there are too few massive systems at such a large redshift to make a fair comparison based on the X-ray morphology.

Having built the associations between simulated and real clusters, we estimate the concentrations by averaging over the selected projections. The results are listed in the sixth column of Table 3 for the NFW model. In the fifth column, we report the mass range of the selected halos. On the basis of these results, we find that CLASH-like clusters have concentrations in the range $\sim[3-6]$. These measurements are shown in Figure 17. The different colors allow one to discriminate between the redshifts of the simulations. For comparison, we also show the $c-M-z$ relation previously determined using the larger sample of X-ray-selected halos.

The X-ray morphology may reflect the orientation of the cluster. Clusters may appear to have round X-ray isophotes if they have prolate three-dimensional shapes and have their major axis aligned with the line of sight. Knowing the shapes and orientation of the MUSIC-2 halos, we can estimate whether a sample constructed to resemble the morphology of the CLASH clusters is likely to be affected by a large orientation bias. On the basis of the associations we made between real and simulated clusters, we find that the mean angle between the major axes of the simulated halos and the line of sight is $\sim 54 \mathrm{deg}$. This indicates that the orientation bias is modest because the expected angle for a distribution of random orientations is $\sim 57 \mathrm{deg}$.

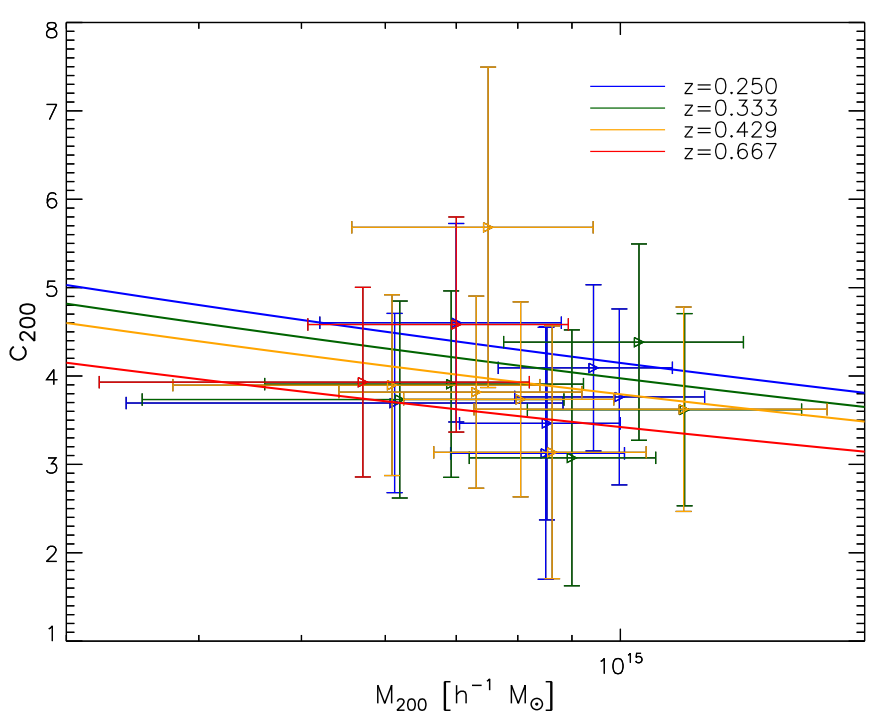

Figure 17. NFW concentrations and masses of MUSIC-2 halos matching the X-ray morphologies of the CLASH X-ray-selected clusters. The error bars reflect the scatter in the masses and concentrations of the halos matching each CLASH cluster. The data points have different colors depending on the redshift of the simulations. For comparison, we also show the $c-M-z$ relation derived from the simulated X-ray-selected sample, whose parameters are given in Table 2 (solid lines).

(A color version of this figure is available in the online journal.)

\section{SUMMARY AND CONCLUSIONS}

In this paper, we used a large set of 1,419 cluster-sized halos evolved in $N$-body/hydrodynamical simulations and distributed over the redshift range $0.25 \leqslant z \leqslant 0.67$ to make predictions about several properties of the clusters included in the CLASH sample (Postman et al. 2012). The simulations used here, which are taken from the MUSIC-2 sample (Sembolini et al. 2013b), intentionally do not include radiative physics in order to avoid an artificial boost of the halo concentrations due to the well-known overcooling problem.

First, we characterized the halos by studying their total density profiles. We fitted the profiles using three fitting models: the NFW, the gNFW, and the Einasto profiles. We derived concentration-mass relations and we quantified their dependence on the degree of relaxation. By fitting with the gNFW and Einasto profiles, we could also investigate the distribution of the inner slopes and of the shape parameters of the density profiles.

We combined our work with the measurements of concentrations and masses taken from J. Vega et al. (in preparation). These measurements were obtained by fitting the surface-density profiles extracted from hundreds of projections of the MUSIC-2 halos. The fits were performed with the same codes used to measure the surface-density profiles recovered from the strong and weak lensing analyses of the CLASH cluster sample, as described in Merten et al. (2014). The radial ranges over which the fits were performed are compatible with those used in the observational analysis.

Using the X-MAS code (Gardini et al. 2004; Rasia et al. 2011), we produced simulated Chandra observations for three orthogonal lines of sight to each halo above the MUSIC-2 mass completeness limit. These simulated observations were processed using the same routines employed in Donahue et al. (2014) to carry out the X-ray morphological analysis of the CLASH clusters. The X-ray morphology of the simulated halos 
was quantified by means of five morphological parameters, which we combined to define a global regularity parameter.

Using the concentrations and masses derived from the analysis of the surface-density profiles, we derived lensing-like concentration-mass relations, including the effects of selection functions aimed at reproducing some observational properties of the CLASH clusters. In particular, we focused on their ability to produce strong lensing effects and their X-ray regularity. For this purpose, we created two subsamples of MUSIC- 2 halos. The first includes halos with Einstein radii in the range of those of the CLASH clusters. The second is constructed so as to reproduce the distribution of X-ray regularity parameters of the CLASH clusters.

Our results can be summarized as follows.

1. We find that a large fraction of MUSIC-2 halos has density profiles that are better fitted by gNFW and Einasto profiles than by NFW profiles. Not surprisingly, the halos that most deviate from the NFW model are the least relaxed. For these halos, more flexible profiles are needed to better reproduce the shape of the density profiles. The analysis based on the gNFW model shows that the inner slopes of these profiles are distributed over a wide range of values. On average, the logarithmic inner slope is largely consistent with the NFW slope, though. The Einasto profile fits the halos slightly better than does the gNFW model.

2. When seen in projection, the distribution of the inner slopes widens further, and a large fraction of halos is fitted with profiles that are flatter than the NFW at small radii. On average, the inner logarithmic slopes derived from the gNFW fits of the surface-density profiles are $\sim 15 \%$ smaller than found fitting the density profiles. About $15 \%$ of the halos have inner logarithmic slopes smaller than 0.5.

3. The masses derived from the fits of the density profiles match quite well with the true masses of the halos, with a scatter of only a few percent. When they are recovered from the projected mass distributions, mimicking the results obtainable from the analysis of surface-density fields reconstructed via lensing, the masses are smaller than the true masses by less than $5 \%$ on average. As discussed in Giocoli et al. (2012a), a mass bias is expected for randomly oriented, prolated triaxial halos. However, the amplitude of the bias for this sample is $\sim 50 \%$ smaller than expected from semianalytical calculations. The bias is even smaller for relaxed halos because their shapes are more spherical.

4. The concentrations derived from the fits of the density profiles with different models are rather similar. However, we find that Einasto concentrations are smaller by $10-15 \%$ compared to the NFW and gNFW concentrations.

5. We find that the MUSIC-2 halos follow an intrinsic concentration-mass relation characterized by a slightly larger normalization compared to other concentration-mass relations recently proposed in the literature for the NFW model. The redshift evolution is rather weak.

6. When we mimic the selection of clusters on the basis of their strong lensing signal, we find that the concentration-mass relation derived from the analysis of the projected mass distributions is considerably steeper than expected for nonstrong lenses. It also has a larger normalization. This result holds for all of the fitting models used in this work.

7. Using the X-ray regularity parameter $M$ to select halos with regular X-ray morphologies leads to the inclusion of both relaxed and unrelaxed halos in the sample. Therefore, the $\mathrm{X}$-ray morphology, especially if evaluated in a relatively small region around the cluster center, is not ideal for identifying relaxed halos.

8. The parameter $M$ is correlated with the halo $2 \mathrm{D}$ concentration. The most regular halos have higher mass concentrations compared to the full sample of simulated halos because they could be measured from a lensing analysis. The excess of concentration is explained in terms of (1) the higher fraction of super-relaxed objects in the X-rayselected sample and (2) the presence, among the selected halos, of unrelaxed systems that happen to be well aligned with the line of sight. For a regularity parameter $M$ equal to the median value measured for the CLASH sample, we expect that the concentration will be higher than the average of all halos in the simulated set by $\sim 11 \% \pm 3 \%$.

9. Measuring the concentration-mass relation and its redshift evolution in a subsample of MUSIC-2 halos that reproduces the distribution of X-ray regularity parameters of the clusters in the CLASH X-ray-selected sample, we find that this has an amplitude and mass dependence similar to those of the concentration-mass relation of strong lensing clusters. We verified that the sample of X-ray-selected halos is largely composed of strong lensing clusters and contains a fraction of only $8 \%$ of halos that do not have extended critical lines for sources at $z \sim 2$.

10. The sample of X-ray-selected halos is in large part composed of relaxed halos. These amount to $\sim 70 \%$ of the sample.

These results suggest that the CLASH clusters are prevalently relaxed and likely to be modestly affected by strong lensing bias. Once accounting for projection and selection effects, their NFW concentrations are expected to scale with mass as $c \propto M^{-0.16 \pm 0.11}$ for the NFW model, resulting in average concentrations that are intermediate between those predicted in $3 \mathrm{D}$ for relaxed and super-relaxed halos in the mass range $2 \times 10^{14} \lesssim M_{200} \lesssim 10^{15} h^{-1} M_{\odot}$. Matching the simulations to the individual CLASH clusters on the basis of the X-ray morphology, we expect that the NFW concentrations recovered from the lensing analysis of the CLASH clusters are in the range [3-6], with an average value of 3.87 and a standard deviation of 0.61 . The median value of the concentrations in the simulated sample is 3.76 , and the first and third quartiles of the concentration distribution are 3.62 and 3.93, respectively. As shown in Meneghetti et al. (2010a) and in Hennawi et al. (2008), strong lensing clusters are expected to be frequently elongated along the line of sight. For the simulated CLASH sample, the median angle between the major axis of the halos and the lines of sight selected from the X-ray analysis is $54 \mathrm{deg}$. This indicates that the orientation bias is very modest. It is consistent with the results based on the analysis of the halos from the MareNostrum Universe presented in Meneghetti et al. (2010a).

The research was in part carried out at the Jet Propulsion Laboratory, California Institute of Technology, under a contract with the National Aeronautics and Space Administration. M.M. thanks ORAU and NASA for supporting his research at JPL. M.M., C.G., and L.M. acknowledge support from the contracts ASI/INAF I/023/12/0, INFN/PD51, and the PRIN MIUR 2010-2011 "The dark universe and the cosmic evolution of baryons: From current surveys to Euclid.” E.R. acknowledges support from the National Science Foundation AST-1210973, SAO TM3-14008X (issued under NASA Contract No. NAS803060). C.G.'s research is part of the project GLENCO, funded under the European Seventh Framework Programme, Ideas, 
grant agreement No. 259349. K.U. acknowledges support from the National Science Council of Taiwan (grant NSC100-2112M-001-008-MY3) and from the Academia Sinica Career Development Award. Support for A.Z. is provided by NASA through Hubble Fellowship grant \#HST-HF-51334.01-A awarded by STScI. D.G., S.S., and P.R. were supported by SFBTransregio 33 The Dark Universe by the Deutsche Forschungsgemeinschaft (DFG) and the DFG cluster of excellence Origin and Structure of the Universe. This work was supported in part by contract research "Internationale Spitzenforschung II/2-6" of the Baden Württemberg Stiftung. The Dark Cosmology Centre is funded by the DNRF. J.S. was supported by NSF/AST1313447, NASA/NNX11AB07G, and the Norris Foundation CCAT Postdoctoral Fellowship. The MUSIC simulations were performed at the Barcelona Supercomputing Center (BSC), and the initial conditions were done at the Leibniz Rechenzentrum Munich (LRZ). G.Y. and F.S. acknowledge support from MINECO under research grants AYA2012-31101, FPA2012-34694, and MultiDark CSD2009-00064. We thank Stefano Borgani and the whole computational astrophysics group at the University of Trieste and at INAF-OATS for giving us access to their set of hydrodynamical simulations.

\section{REFERENCES}

Allen, S. W., Rapetti, D. A., Schmidt, R. W., et al. 2008, MNRAS, 383, 879 Anders, E., \& Grevesse, N. 1989, GeCoA, 53, 197

Balestra, I., Vanzella, E., Rosati, P., et al. 2013, A\&A, 559, L9

Balmès, I., Rasera, Y., Corasaniti, P.-S., \& Alimi, J.-M. 2014, MNRAS, 437, 2328

Bayliss, M. B., Johnson, T., Gladders, M. D., Sharon, K., \& Oguri, M. 2014, ApJ, 783,41

Becker, M. R., \& Kravtsov, A. V. 2011, ApJ, 740, 25

Bhattacharya, S., Habib, S., Heitmann, K., \& Vikhlinin, A. 2013, ApJ, 766, 32

Biffi, V., Sembolini, F., De Petris, M., et al. 2014, MNRAS, 439, 588

Biviano, A., Rosati, P., Balestra, I., et al. 2013, A\&A, 558, A1

Bonafede, A., Dolag, K., Stasyszyn, F., Murante, G., \& Borgani, S. 2011, MNRAS, 418, 2234

Borgani, S., \& Kravtsov, A. 2011, ASL, 4, 204

Bouwens, R., Bradley, L., Zitrin, A., et al. 2014, ApJ, 795, 126

Bradley, L. D., Zitrin, A., Coe, D., et al. 2014, ApJ, 792, 76

Bryan, G. L., \& Norman, M. L. 1998, ApJ, 495, 80

Buote, D. A., \& Canizares, C. R. 1992, ApJ, 400, 385

Buote, D. A., \& Tsai, J. C. 1996, ApJ, 458, 27

Cassano, R., Ettori, S., Giacintucci, S., et al. 2010, ApJL, 721, L82

Coe, D., Umetsu, K., Zitrin, A., et al. 2012, ApJ, 757, 22

Coe, D., Zitrin, A., Carrasco, M., et al. 2013, ApJ, 762, 32

De Boni, C., Ettori, S., Dolag, K., \& Moscardini, L. 2013, MNRAS, 428, 2921

Despali, G., Tormen, G., \& Sheth, R. K. 2013, MNRAS, 431, 1143

Diemer, B., \& Kravtsov, A. V. 2014, arXiv:1407.4730

Dolag, K., Bartelmann, M., Perrotta, F., et al. 2004, A\&A, 416, 853

Donahue, M., Voit, G., Mark, M. A., et al. 2014, ApJ, 794, 136

Dubinski, J., \& Carlberg, R. G. 1991, ApJ, 378, 496

Duffy, A. R., Schaye, J., Kay, S. T., \& Dalla Vecchia, C. 2008, MNRAS, 390, L64

Duffy, A. R., Schaye, J., Kay, S. T., et al. 2010, MNRAS, 405, 2161

Dutton, A. A., \& Macciò, A. V. 2014, arXiv:1402.7073

Ebeling, H., Barrett, E., Donovan, D., et al. 2007, ApJL, 661, L33

Einasto, J., \& Haud, U. 1989, A\&A, 223, 89

Fabjan, D., Borgani, S., Tornatore, L., et al. 2010, MNRAS, 401, 1670

Frenk, C. S., White, S. D. M., Davis, M., \& Efstathiou, G. 1988, ApJ, 327, 507

Gao, L., Navarro, J. F., Frenk, C. S., et al. 2012, MNRAS, 425, 2169

Gardini, A., Rasia, E., Mazzotta, P., et al. 2004, MNRAS, 351, 505

Giocoli, C., Meneghetti, M., Ettori, S., \& Moscardini, L. 2012a, MNRAS, 426,1558

Giocoli, C., Meneghetti, M., Metcalf, R. B., Ettori, S., \& Moscardini, L. 2013, arXiv: 1311.1205

Giocoli, C., Tormen, G., \& Sheth, R. K. 2012b, MNRAS, 422, 185
Gralla, M. B., Sharon, K., Gladders, M. D., et al. 2011, ApJ, 737, 74

Gruen, D., Brimioulle, F., Seitz, S., et al. 2013, MNRAS, 432, 1455

Hennawi, J. F., Dalal, N., Bode, P., \& Ostriker, J. P. 2007, ApJ, 654, 714

Hennawi, J. F., Gladders, M. D., Oguri, M., et al. 2008, AJ, 135, 664

Jing, Y., \& Suto, Y. 2002, ApJ, 574, 538

Killedar, M., Borgani, S., Meneghetti, M., et al. 2012, MNRAS, 427, 533

Klypin, A., Kravtsov, A. V., Bullock, J. S., \& Primack, J. R. 2001, ApJ, 554,903

Kravtsov, A., \& Borgani, S. 2012, arXiv:1205.5556

Kravtsov, A. V., Klypin, A. A., \& Khokhlov, A. M. 1997, ApJS, 111, 73

Lemze, D., Postman, M., Genel, S., et al. 2013, ApJ, 776, 91

Lemze, D., Sadeh, S., \& Rephaeli, Y. 2009, MNRAS, 397, 1876

Lemze, D., Wagner, R., Rephaeli, Y., et al. 2012, ApJ, 752, 141

Limousin, M., Morandi, A., Sereno, M., et al. 2013, SSRv, 177, 155

Ludlow, A. D., Navarro, J. F., Angulo, R. E., et al. 2013, arXiv:1312.0945

Ludlow, A. D., Navarro, J. F., Li, M., et al. 2012, arXiv:1206.1049

McCarthy, I. G., Schaye, J., Bower, R. G., et al. 2011, MNRAS, 412, 1965

Medezinski, E., Umetsu, K., Nonino, M., et al. 2013, ApJ, 777, 43

Meneghetti, M., Bartelmann, M., Dahle, H., \& Limousin, M. 2013, SSRv, 177,31

Meneghetti, M., Fedeli, C., Pace, F., Gottlöber, S., \& Yepes, G. 2010a, A\&A, 519, A90

Meneghetti, M., \& Rasia, E. 2013, arXiv:1303.6158

Meneghetti, M., Rasia, E., Merten, J., et al. 2010b, A\&A, 514, A93

Merritt, D., Graham, A. W., Moore, B., Diemand, J., \& Terzić, B. 2006, AJ, 132,2685

Merten, J., Cacciato, M., Meneghetti, M., Mignone, C., \& Bartelmann, M. 2009, A\&A, 500, 681

Merten, J., Coe, D., Dupke, R., et al. 2011, MNRAS, 417, 333

Merten, J., Meneghetti, M., Postman, M., et al. 2014, arXiv:1404.1376

Moore, B., Governato, F., Quinn, T., Stadel, J., \& Lake, G. 1998, ApJL, 499, L5

Muñoz-Cuartas, J. C., Macciò, A. V., Gottlöber, S., \& Dutton, A. A. 2011, MNRAS, 411, 584

Nakamura, T. T., \& Suto, Y. 1997, PThPh, 97, 49

Navarro, J. F., Frenk, C., \& White, S. 1996, ApJ, 462, 563

Navarro, J. F., Frenk, C., \& White, S. 1997, ApJ, 490, 493

Navarro, J. F., Ludlow, A., Springel, V., et al. 2010, MNRAS, 402, 21

Neto, A. F., Gao, L., Bett, P., et al. 2007, MNRAS, 381, 1450

Newman, A. B., Treu, T., Ellis, R. S., \& Sand, D. J. 2011, ApJL, 728, L39

Oguri, M., Bayliss, M. B., Dahle, H., et al. 2012, MNRAS, 420, 3213

Oguri, M., \& Blandford, R. D. 2009, MNRAS, 392, 930

Oguri, M., Hennawi, J. F., Gladders, M. D., et al. 2009, ApJ, 699, 1038

Planelles, S., Borgani, S., Fabjan, D., et al. 2014, MNRAS, 438, 195

Postman, M., Coe, D., Benítez, N., et al. 2012, ApJS, 199, 25

Rasia, E., Borgani, S., Ettori, S., Mazzotta, P., \& Meneghetti, M. 2013a, ApJ, 776,39

Rasia, E., Mazzotta, P., Evrard, A., et al. 2011, ApJ, 729, 45

Rasia, E., Meneghetti, M., \& Ettori, S. 2013b, AstRv, 8, 010000

Rasia, E., Meneghetti, M., Martino, R., et al. 2012, NJPh, 14, 055018

Redlich, M., Bartelmann, M., Waizmann, J.-C., \& Fedeli, C. 2012, A\&A, 547, A66

Retana-Montenegro, E., van Hese, E., Gentile, G., Baes, M., \& Frutos-Alfaro, F. 2012, A\&A, 540, A70

Sarazin, C. L. 1986, RvMP, 58, 1

Schneider, P., Ehlers, J., \& Falco, E. E. 1992, Gravitational Lenses (Berlin: Springer)

Sembolini, F., De Petris, M., Yepes, G., et al. 2013a, arXiv:1309.5387

Sembolini, F., Yepes, G., De Petris, M., et al. 2013b, MNRAS, 429, 323

Skibba, R. A., \& Macciò, A. V. 2011, MNRAS, 416, 2388

Skibba, R. A., van den Bosch, F. C., Yang, X., et al. 2011, MNRAS, 410, 417

Smit, R., Bouwens, R. J., Labbe, I., et al. 2013, arXiv:1307.5847

Springel, V. 2005, MNRAS, 364, 1105

Torri, E., Meneghetti, M., Bartelmann, M., et al. 2004, MNRAS, 349, 476

Umetsu, K., Medezinski, E., Nonino, M., et al. 2012, ApJ, 755, 56

Umetsu, K., Medezinski, E., Nonino, M., et al. 2014, arXiv:1404.1375

Zhao, D. H., Jing, Y. P., Mo, H. J., \& Börner, G. 2009, ApJ, 707, 354

Zhao, H. 1996, MNRAS, 278, 488

Zheng, W., Postman, M., Zitrin, A., et al. 2012, Natur, 489, 406

Zitrin, A., Broadhurst, T., Coe, D., et al. 2011, ApJ, 742, 117

Zitrin, A., Fabris, A., Merten, J., et al. 2014, arXiv:1411.1414

Zitrin, A., Meneghetti, M., Umetsu, K., et al. 2013, ApJL, 762, L30

Zitrin, A., Moustakas, J., Bradley, L., et al. 2012a, ApJL, 747, L9

Zitrin, A., Rosati, P., Nonino, M., et al. 2012b, ApJ, 749, 97 